\title{
Angiogenesis and vascular remodelling in normal and cancerous tissues
}

\author{
Markus R. Owen • Tomás Alarcón • \\ Philip K. Maini • Helen M. Byrne
}

Received: 28 June 2007 / Revised: 2 March 2008 / Published online: 22 October 2008

(C) Springer-Verlag 2008

\begin{abstract}
Vascular development and homeostasis are underpinned by two fundamental features: the generation of new vessels to meet the metabolic demands of under-perfused regions and the elimination of vessels that do not sustain flow. In this paper we develop the first multiscale model of vascular tissue growth that combines blood flow, angiogenesis, vascular remodelling and the subcellular and tissue scale dynamics of multiple cell populations. Simulations show that vessel pruning, due to low wall shear stress, is highly sensitive to the pressure drop across a vascular network, the degree of pruning increasing as the pressure drop increases. In the model, low tissue oxygen levels alter the internal dynamics of normal cells, causing them to release vascular endothelial growth factor (VEGF), which stimulates angiogenic sprouting.
\end{abstract}

Electronic supplementary material The online version of this article (doi:10.1007/s00285-008-0213-z) contains supplementary material, which is available to authorized users.

M. R. Owen $(\varangle) \cdot$ H. M. Byrne

School of Mathematical Sciences, University of Nottingham, Nottingham NG7 2RD, UK

e-mail: markus.owen@nottingham.ac.uk

H. M. Byrne

e-mail: helen.byrne@nottingham.ac.uk

T. Alarcón

Institute for Mathematical Sciences, Imperial College London, London SW7 2PG, UK

e-mail: a.tomas@imperial.ac.uk

\section{P. K. Maini}

Centre for Mathematical Biology, University of Oxford, Oxford OX1 3LB, UK

e-mail: maini@maths.ox.ac.uk

P. K. Maini

Oxford Centre for Integrative Systems Biology, University of Oxford, Oxford OX1 3QU, UK 
Consequently, the level of blood oxygenation regulates the extent of angiogenesis, with higher oxygenation leading to fewer vessels. Simulations show that network remodelling (and de novo network formation) is best achieved via an appropriate balance between pruning and angiogenesis. An important factor is the strength of endothelial tip cell chemotaxis in response to VEGF. When a cluster of tumour cells is introduced into normal tissue, as the tumour grows hypoxic regions form, producing high levels of VEGF that stimulate angiogenesis and cause the vascular density to exceed that for normal tissue. If the original vessel network is sufficiently sparse then the tumour may remain localised near its parent vessel until new vessels bridge the gap to an adjacent vessel. This can lead to metastable periods, during which the tumour burden is approximately constant, followed by periods of rapid growth.

Keywords Blood flow · Multiscale modelling - Tumour angiogenesis · Vascular adaptation · Vascularisation · VEGF

Mathematics Subject Classification (2000) $\quad 9208 \cdot 92 \mathrm{C} 15 \cdot 92 \mathrm{C} 17 \cdot 92 \mathrm{C} 35 \cdot 62 \mathrm{P} 10$

\section{Introduction}

Angiogenesis, the process by which new vessels are generated from the existing vasculature, is generally believed to be a turning point in the development of solid tumours. During the avascular phase that precedes angiogenesis, the growth of the tumour is limited by the rate at which nutrients and metabolites diffuse across the outer tumour boundary. Such avascular tumours undergo diffusion-limited growth and, as a result, are typically at most several millimeters in radius. Following successful angiogenesis, the tumour has its own vascular network which provides access to an almost limitless supply of resources and permits unlimited growth of the tumour mass. The vasculature also provides a route for tumour cells that break free from the primary lesion to metastasise to other parts of the host organism. Thus, angiogenesis marks a tumour's transition from a localised and relatively harmless lesion to a systemic and potentially fatal disease [21].

Angiogenesis is a highly coordinated, yet extremely complex, process which involves degradation of the extracellular matrix, endothelial cell (EC) migration and proliferation, loop formation (anastomosis) by capillary sprouts, vessel maturation, and blood flow [43]. Further pruning and remodelling of the vascular network may be stimulated by tissue-derived signalling molecules and blood flow conditions (e.g. wall-shear stress and pressure) $[19,42]$. The initiating event in tumour-induced angiogenesis is the secretion of tumour angiogenic factors (TAFs), such as vascular endothelial growth factor (VEGF), by tumour cells usually in response to poor oxygenation (hypoxia) in the inner regions of an avascular tissue [22,41]. The TAFs diffuse through the tissue until they reach the existing vasculature. There they stimulate the ECs lining the blood vessels to form sprouts which migrate, via chemotaxis, towards the source of TAF, and to proliferate (for a detailed discussion of angiogenesis see [17,26,41]).

In view of its vital role in tumour growth, targetting angiogenesis as a therapeutic strategy was initially expected to provide a significant breakthrough in cancer therapy. 
The rationale underlying such anti-angiogenic therapy is as follows: reducing the blood supply to a tumour will severely diminish its nutrient supply, causing it either to disappear or to shrink to a harmless, avascular state. A variety of anti-angiogenic compounds have been identified and shown to act directly, by inactivating the TAF molecules, or indirectly, by blocking TAF receptors on ECs. Unfortunately the success of early animal studies has not been repeated in clinical trials where anti-angiogenic agents used in isolation produce only modest increases in life-expectancy and longterm survival (see [27] and references therein). Further investigations have revealed that, when combined with conventional radio- or chemo-therapy, the net effect is considerably better with the anti-angiogenic agents than for radio- or chemo-therapy alone [27]. Teicher [49] hypothesises that the increased response occurs because the combined therapy simultaneously targets two cancer compartments, the tumour and the endothelial cells, rather than one [49].

Within fully vascularised tumours the situation is even more complex. Tumour vessels are usually immature and lack the well-defined anatomical structure of their normal counterparts. Consequently, they are leaky and highly proliferative. Because they are immature, they lack muscular tone and are prone to collapse under the pressure of the growing tumour mass. These structural abnormalities mean that tumour blood flow is often chaotic, with marked spatio-temporal heterogeneity in oxygen delivery. These features create an abnormal microenvironment in which increased hypoxia and aberrant tumour blood flow pose severe barriers to drug delivery and efficacy [25]. The situation is further complicated by the fact that angiogenesis continuously alters the vasculature, making it extremely difficult to estimate, with confidence, the amount of drug being delivered to a tumour. As we explain below, the development of realistic (and validated) mathematical models of vascular tumour growth that account explicitly for angiogenesis has considerable potential for resolving these problems.

In this context, it seems that normalising the tumour vasculature could improve the delivery of drugs and oxygen to tumour regions. This is the mechanism by which certain anti-angiogenic therapies are believed to improve cytotoxic drug efficiency [27, 50,53]. The rationale behind such normalisation strategies can be explained as follows. In pathology and normal physiology, angiogenesis is controlled by the balance between angiogenic promoters (e.g. VEGF) and their inhibitors. In normal conditions, feedback mechanisms ensure that, when the vascular density is sufficient to meet the metabolic demands of the tissue, the inhibitors will eventually dominate and angiogenesis will cease. In pathology, the feedback mechanisms are disrupted and an imbalance in favour of angiogenesis persists. Using anti-angiogenic compounds to restore this balance should, in principle, result in a more normal-looking vasculature. However, several studies report that anti-angiogenic therapies antagonise chemo- or radio-therapy by reducing oxygenation and drug delivery. This suggests that there is a delicate balance between normalisation and (excessive) vessel regression, implying that extreme care is needed in the dose and schedule of normalisation therapies [27].

Interpreting the vast quantity of clinical and experimental data relating to vascular tumour growth and angiogenesis is now creating a strong demand for a systematic approach to studying angiogenesis. Mathematical modelling represents a natural way to combine such data, test existing hypotheses, and generate new ones. As this view becomes more widespread, theoretical modelling of the type presented in this paper 
will play an increasingly important role in improving our understanding of the complex processes that underpin angiogenesis and vascular tissue growth.

There is a long history of modelling angiogenesis in different biological contexts including tumour growth [10], wound healing and placental development, and an equally wide variety of theoretical approaches have been proposed (for a review, see [31]). For example, in [8,9] spatially-averaged models are formulated as systems of coupled ordinary differential equations. Such spatially-averaged models can include considerable detail of the underlying biochemistry but are unable to account for the morphology of the vascular network. The earliest, one-dimensional continuum models of angiogenesis focus on the proliferation and directed motion of populations of ECs towards sources of VEGF and exhibit many experimentally observed features of angiogenesis (e.g. acceleration of the capillary tips as they migrate towards the tumour, with the maximum capillary tip density preceding the maximum vessel density) [16]. However, they, too, are unable to replicate the detailed morphology of evolving vasculature. Stochastic models which track the chemotactic movement of individual capillary tips towards high regions of VEGF can account for details of the evolving vascular network $[7,47]$ and capture many characteristic features of angiogenesis. However, they do not account for blood flow within the vasculature or consider the dynamic interplay between the evolving vasculature and growing tumour mass. Such models also typically focus on a single spatial scale of organisation. As a result, they are unable to explain how phenomena at different scales are coupled or to combine, in a systematic manner, data from the different scales.

More recently several groups have started to develop mathematical models of angiogenesis in which individual vessels form a network that delivers nutrients and drugs to the tissue [11,23]. For example, McDougall and coworkers [32,33,45,46] have developed a hybrid model that focusses on vascular adaptation and angiogenesis, without explicitly considering the tumour's growth. In [33] they showed that the structural abnormality of tumour vasculature could be responsible for the failure of blood-borne drugs to penetrate large regions of the tumour [25]. Bartha et al. [11,28,52] have recently developed a similar approach, which addresses the need to combine tumour growth with vascular remodelling, by considering a growing tumour which coopts an existing normal vasculature. Their simulated vessel networks are remodelled via vessel collapse, angiogenesis in the tumour periphery, and vessel dilation within the tumour.

In a series of papers, Alarcón and coworkers developed a multiscale model of vascular tumour growth that links vascular adaptation, blood flow, oxygen and growth factor transport at the tissue scale to the subcellular and cellular dynamics of normal and cancerous cells [2,5]. The basic framework was introduced in [4] and has since been extended to account for several features, including active cell movement [14], vessel co-option and dematuration and the effect of combination therapy [27]. The main purpose of this paper is to show how this multiscale model can be extended to include angiogenesis. In the previous versions of the model the vasculature was assumed to undergo vascular structural adaptation, dematuration and collapse, but the topology of the vasculature was considered to be static $[4,5]$. This means that new vessels and connections between them were not considered. In the present work this limitation is removed. 
The paper is organised as follows. In Sect. 2 we describe our multiscale model of vascular tumour growth, focussing on the new features, such as angiogenesis and vessel regression, that we have incorporated. Numerical simulations are presented in Sects. 3 and 4. Attention focusses on normal tissue growth and vascular adaptation in Sect. 3 so that we can allow the vascular density to evolve or adapt itself to the metabolic demands on the healthy tissue that it perfuses. We find that vessel pruning is highly sensitive to the pressure drop across the vascular network, the degree of pruning increasing as the pressure drop increases. Since angiogenesis is stimulated by VEGF, which is produced under hypoxia, we find that the level of blood oxygenation regulates the extent of angiogenesis and the mean long term vascular density, with higher oxygenation leading to fewer vessels. We also find that the initial vascular architecture can affect its final density, particularly when the pressure drop across the network is high enough to support low levels of vessel pruning. Simulations that combine these features show that network remodelling is best achieved via an appropriate balance between pruning and angiogenesis, in the sense that an optimal network oxygenates the tissue area with the lowest possible vascular density. In Sect. 4 we assess the impact of introducing a small cluster of mutant cells into the tissue. Tumour growth is accompanied by the formation of hypoxic regions and the production of VEGF, stimulating angiogenic sprouting, and causing the vascular density to exceed that for normal tissue. We also find that if the original vessel network is sufficiently sparse, then the tumour can remain localised near to isolated parent vessels until new vessels bridge the gap to an adjacent vessel. The paper concludes in Sect. 5 with a summary of our findings and a discussion of directions for future research.

\section{Model description}

We summarise below the main features of our multiscale model, starting in Sect. 2.1 with a description of the original model of vascular tumour growth on which it is based before explaining in Sect. 2.2 how angiogenesis is incorporated. The interested reader is referred to references $[4,5,15]$ for more detailed information about the basic model. In addition, Appendices A and B include further details of the model and a full statement of the parameter values.

\subsection{General model framework}

Our basic model integrates phenomena occurring on markedly different time and length scales. These features include blood flow and structural adaptation of the vascular network, transport within the tissue of blood-borne oxygen, cell division and apoptosis, competition between the normal and cancerous cells for space and for vital nutrients such as oxygen, and the expression by the cells of growth factors such as VEGF, a potent angiogenic factor. Our theoretical framework is based on the hybrid cellular automaton concept which has been used to model many different aspects of tumour development (see [2,6,20,37]). As in [2], the model that we develop in this paper accounts not only for species that diffuse through the tissue (here oxygen and VEGF), but also for subcellular and cell-scale phenomena, and the coupling 


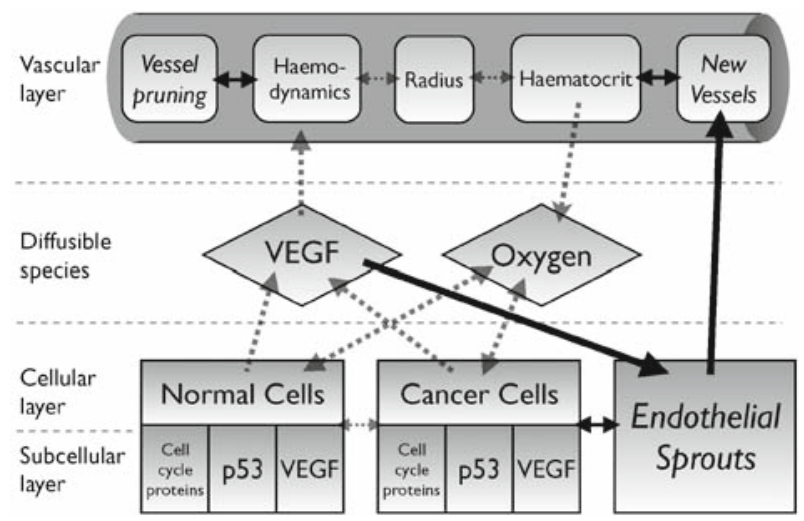

Fig. 1 The structure of our multiscale model of vascular tumour growth. In this paper we introduce VEGFinduced endothelial cell sprouting, leading to new vessel formation. In addition, vessels do not survive if their flow is too low for too long. The solid arrows indicate these new mechanisms, and dashed arrows those mechanisms outlined previously $[4,5,15]$

between them. To this end, our model is organised into three layers, which correspond, respectively, to the tissue (or vascular), cellular and subcellular time and length scales (see Fig. 1).

The vascular layer Here we focus on the structure of the network and blood flow (see Appendix A.3 and [2,4,5,15] for more details). In an extension to our previous work which focussed on hexagonal networks of blood vessels, with constant topology, we now consider an arbitrary vascular network in which individual vessels undergo structural adaptation (i.e. changes in their radii and haematocrit) in response to a variety of stimuli. According to the structural adaptation law, the radius of a vessel at time $t, R(t)$, is updated with timestep $\varepsilon_{t}$ according to:

$$
R\left(t+\varepsilon_{t}\right)=R(t)+\varepsilon_{t} R(t)\left(S_{h}+S_{m}+S_{d}+S_{u}-k_{s}\right),
$$

subject to $R_{\mathrm{MIN}} \leq R\left(t+\varepsilon_{t}\right) \leq R_{\mathrm{MAX}}$, and where

$$
S_{h}=\log \left(\tau_{w}+\tau_{\text {ref }}\right)-k_{p} \log (\tau(P))
$$

is the haemodynamic stimulus,

$$
S_{m}=k_{m}(V) \log \left(\frac{\dot{Q}_{\mathrm{ref}}}{\dot{Q} H}+1\right)
$$

is the metabolic stimulus, and $k_{s}$ is the shrinking stimlus. $S_{d}$ and $S_{u}$ represent the downstream and upstream components of the conducted stimulus [40], which was previously introduced into our multiscale model [5] - however, for simplicity, and to avoid obscuring the main issues (angiogenesis and vessel pruning), we neglect them here. 
In Eqs. (1)-(3), $\dot{Q}$ is the flow rate, $\dot{Q}_{\text {ref }}, k_{m}$ and $k_{s}$ are (non-negative) constants, $H$ is the haematocrit (fractional volume of red blood cells), $\tau_{w}=R \Delta P / L$ is the wall shear stress acting on a vessel of length $L$ and $P$ is the transmural pressure. The magnitude of the corresponding "set point" value of the wall shear stress, $\tau(P)$, is obtained from an empirical fit to experimental data (see Eq. (22) in Appendix A.3). In [4] coupling to the subcellular level was introduced by assuming that $k_{m}=k_{m}(V)$ where $V$ denotes the local, extracellular VEGF concentration. To implicitly account for the pro-angiogenic impact of VEGF, $k_{m}(V)$ was assumed to be an increasing saturating function, of the form:

$$
k_{m}(V)=k_{m}^{0}\left(1+k_{m}^{V} \frac{V}{V_{0}+V}\right)
$$

for constants $k_{m}^{0}, k_{m}^{V}$ and $V_{0}$. In the following, except for comparison purposes in Sect. 4 , we set $k_{m}^{V}=0$ since we replace this implicit angiogenesis with explicit growth of new vessels.

In addition to determining vessel radii we also compute the blood flow rate, the pressure drop and the haematocrit distribution in each vessel. These in turn affect the vessel radii, forming a nonlinear feedback loop which is iterated until the maximum relative change in radius across the network is less than some tolerance, $R_{\text {reltol }}$ (see Appendix A.3). Thus we treat vessel radii, haematocrits and flows as being at quasisteady state with respect to the other layers of the multiscale model. Previously, we have implemented flow-dependent haematocrit splitting at branch points according to Yen and Fung [54]. Here we assume symmetric splitting in order to focus on the dynamics of remodelling via pruning and angiogenesis-we revisit this issue in the discussion.

The diffusible layer Indirect coupling between the vascular and cellular layers is achieved by the diffusive transport of oxygen and VEGF within the tissue, reactiondiffusion equations being used to model the spatio-temporal evolution of both species. For oxygen, $C(\mathbf{x})$, the blood-borne haematocrit within each branch of the vascular network acts as a distributed source while the cells act as spatially-distributed sinks. We combine these features in the following dimensionless partial differential equation (employing the usual adiabatic assumption):

$$
0=D_{c} \nabla^{2} C+2 \pi R(\mathbf{x}) \mathscr{P}_{c}\left(C_{\text {blood }}-C\right)-k(\mathbf{x}) C,
$$

where $D_{c}$ is the oxygen diffusion coefficient, $R(\mathbf{x})$ is the vessel radius if there is one at position $\mathbf{x}$ and is zero otherwise, $\mathscr{P}_{c}$ is the vessel permeability to oxygen, $C_{\text {blood }}$ is the blood oxygen level (assumed proportional to the vessel haematocrit), and $k(\mathbf{x})$ is a consumption rate per cell which depends on the cell type at position $\mathbf{x}$. A similar equation is used to update the extracellular concentration of VEGF, $V(\mathbf{x})$, the cells now acting as sources and the vessels as sinks,

$$
0=D_{v} \nabla^{2} V+k_{v}(\mathbf{x})-2 \pi R(\mathbf{x}) \mathscr{P}_{v} V-\delta_{v} V
$$


Here, $D_{v}$ is the VEGF diffusion coefficient, $k_{v}(\mathbf{x})$ is a cell type dependent VEGF secretion rate, $\mathscr{P}_{v}$ is the vessel permeability to VEGF, $\delta_{v}$ is the rate of VEGF decay, and VEGF uptake by vessels assumes $V_{\text {blood }}=0$. Normal cells are assumed to only release VEGF if their internal VEGF level is greater than a threshold, $V_{\text {THR }}$.

The cellular layer In the cellular layer, we concentrate on cell-cell interactions (competition) between the normal and cancerous cells. The two cell types compete for space and resources, the cancerous phenotype usually outperforming its normal counterpart. Competition between the two cell types is introduced by simple rules, which connect the cellular and subcellular layers. For example, apoptosis (programmed cell death) in normal cells is controlled by the expression of p53 (whose dynamics are dealt with in the subcellular layer): if the level of p53 in a normal cell exceeds a threshold value then the cell undergoes apoptosis. This threshold level is high $\left(p 53_{\mathrm{THR}}^{\text {high }}\right.$ ) if the ratio of normal cells to cancer cells in the neighbourhood of a normal cell is sufficiently high, otherwise the p53 threshold for normal cell apoptosis is set to $p 53_{\mathrm{THR}}^{\text {low }}$. In this way, normal cells are more likely to undergo apoptosis at the edge of or within a tumour, providing a secondary mechanism for tumour invasion.

Note that, following [14], each element of the cellular automaton may contain more than one cell, with each cell type having its own carrying capacity for cell division $\left(D_{m}\right)$ and movement $\left(N_{m}\right)$. In the following we set these carrying capacities to be one for both normal and cancer cells, so that each element can contain at most one normal or cancer cell. However, we will set the carrying capacity for movement of endothelial tip cells to be greater than one, enabling them to move to locations that are already occupied. Movement is via a random walk, similar to that described in [14] —each active cell in a simulation is given a chance to move, in an order which is randomised in each time step. Section 2.2 below defines the transition probabilities, including a bias due to chemotactic gradients.

The subcellular layer Processes that are modelled here include the cell cycle, apoptosis, and VEGF secretion, with ordinary differential equations (ODEs) being used to describe the relevant biochemistry. An important and distinguishing feature of our multiscale model is the way in which the dynamics of the intracellular phenomena are regulated by (and regulate) extracellular factors such as oxygen and VEGF levels. In particular, the cell division rate is an increasing function of extracellular oxygen concentration, via the oxygen dependent degradation of the cell-cycle protein $\mathrm{p} 27$. In contrast, the expression of p53, which regulates apoptosis, and VEGF increases under hypoxia. Since the spatial distribution of oxygen depends on the spatial distribution of cells (cellular layer) and haematocrit (vascular layer), processes at the subcellular level are intimately linked to the behaviour of the other two layers: cell proliferation and apoptosis alter the spatial distribution of the cells (see Fig. 1); in addition, vascular remodelling is indirectly regulated by the cellular and intracellular layers via VEGF which is released from the cells, diffuses through the tissue and acts on the endothelial cells (ECs) lining the vessels. We refer the reader to Appendix A.1 for further details of the ordinary differential equations representing the subcellular layer. 
New model features Before explaining in the next section how we explicitly account for angiogenesis, we conclude this section by highlighting other features which are new to our original multiscale model. First, our model can now simulate arbitrary vascular networks. Second, the rate of oxygen delivery from a blood vessel depends on its haematocrit and its surface area (note that if the flow in a vessel is zero then we set the haematocrit in that vessel to zero and the vessel cannot deliver oxygen to the surrounding tissue). Thirdly, we account for vessel pruning or regression. One of the earliest indications that low wall shear stress leads to vessel pruning is due to Clark [19], who observed that small blood vessels tend to regress after a decrease in flow. More recent evidence suggests that this is initiated via apoptosis of endothelial cells $[34,42]$. To model this process, if the wall shear stress in a vessel falls below a threshold value, $\tau_{w}^{\text {crit }}$, then a timer starts. We chose this threshold to be an order of magnitude less than the typical WSS seen in our basic simulations. The timer is only reset if the wall shear stress rises back above $\tau_{w}^{\text {crit }}$. If the timer remains active for longer than a prescribed period, $T_{\text {prune }}$ (i.e. the time the vessel can survive with impaired blood flow), then that branch is removed from the vascular network.

We remark that removing vessels from the network alters its topology and could (in principle) lead to the formation of blunt-ended vessels. If this happens then the resulting blunt-ended vessels will be pruned in the same way (since they cannot support blood flow) unless they establish new connections that permit flow. Finally we remark that in our model the dynamics of the normal and cancerous cells are tightly coupled to the vascular dynamics, a feature which distinguishes our model from the hybrid models of vascular adaptation developed by McDougall and coworkers [18,32,33,45,46].

\subsection{Angiogenesis submodel}

As mentioned in Sect. 1, the main aim of this paper is to extend our multiscale model to include angiogenesis. As in our original multiscale model, VEGF is expressed and secreted by hypoxic cells [4] and diffuses through the tissue. Extracellular VEGF stimulates the ECs that line existing vessels to form blunt-ended capillary sprouts whose tip cells migrate via chemotaxis up spatial gradients in VEGF, new ECs being produced by mitosis at the precise rate necessary to maintain a contiguous sprout between the capillary tip cell and its parent vessel. ECs contained within such bluntended vessels will not experience any flow. As such their wall shear stress will be zero. As for the circulating vasculature, we assume that capillary sprouts can withstand this lack of flow for only a finite period of time: failure to form a connection before this period has elapsed results in regression of the entire sprout. When a tip cell anastomoses with another vessel (or sprout) then the new loop is included in the vascular network.

In more detail, the algorithm that we implement to simulate angiogenesis proceeds as follows:

- In each time step $\Delta t$, and for each lattice site $i$ occupied by a vessel, new sprouts are formed with a probability, $P_{\text {sprout }}$, that depends on the local concentration of VEGF via 


$$
P_{\text {sprout }}=\Delta t \frac{P_{\text {sprout }}^{\max } V}{V_{\text {sprout }}+V}
$$

where $P_{\text {sprout }}=0$ if the number of cells at site $i$ exceeds $E_{m}$. Thus $E_{m}$ characterises the space required for sprout emergence. Around the base of each sprout, we define a radius of exclusion, $R_{\mathrm{ex}}$, within which the formation of another sprout is not allowed (i.e. if there is another sprout nearby, $P_{\text {sprout }}=0$ ). In practice, lateral inhibition via Delta-Notch signalling is thought to regulate the spacing of sprouts-ECs adjacent to those from which sprouts emerge are inhibited from sprouting $[1,24,44,48]$.

- Sprouts are initially labelled as INACTIVE, and retain this label until they connect to another sprout or vessel when they become ACTIVE. There is no flow in INACTIVE sprouts.

- Each sprout is created with AGE $=0$ and this is incremented by $\Delta t$ at each time step.

- The tip of each sprout performs a random walk, biased towards regions of high VEGF concentration. The model for cell movement extends that used in [14] where cell movement is stochastic and the transition probabilities depend on the numbers of cells in a compartment and adjacent compartments. Here we add a dependence on the VEGF gradient between sites. If $N_{m}$ is the carrying capacity for movement of the cell type attempting to move, $N_{i}$ is the number of cells and $V_{i}$ is the VEGF level at site $i, \gamma$ is the chemotactic sensitivity, and $\Omega_{i}$ is the set of sites in a neighbourhood of $i$, not including $i$ itself, then we define $P_{i j}$, the probability of moving from $i$ to $j$ in time $\Delta t$, to be

$$
\begin{aligned}
P_{i j}= & \frac{\Delta t D}{d_{i j}^{2} \Delta x^{2}} \frac{\left(N_{m}-N_{j}\right)}{\sum_{k \in \Omega_{i}}\left(N_{m}-N_{k}\right)+N_{m}-N_{i}+N_{m} M}\left(1+\gamma \frac{V_{j}-V_{i}}{d_{i j} \Delta x}\right) \\
& \text { for } i \neq j,
\end{aligned}
$$

where $d_{i j}$ is the distance in units of $\Delta x$ between sites $i$ and $j$ (and hence is either 1 or $\sqrt{2}$ ) and $D$ is the maximum cell mobility in the absence of chemotaxis. Individual movement probabilities are weighted according to the relative distance moved (this scaling was not used in [14]). The probability of not moving is

$$
P_{i i}=1-\sum_{k \in \Omega_{i}} P_{i k}=1-\frac{\Delta t D}{\Delta x^{2}} \frac{\sum_{k \in \Omega_{i}} \frac{N_{m}-N_{k}}{d_{i k}^{2}}\left(1+\gamma \frac{V_{k}-V_{i}}{d_{i k} \Delta x}\right)}{\sum_{k \in \Omega_{i}}\left(N_{m}-N_{k}\right)+N_{m}-N_{i}+N_{m} M} .
$$

In the limit as $N_{m} \rightarrow \infty$, the mean waiting time is

$$
\Delta t\left(\sum_{k \in \Omega_{i}} P_{i k}\right)^{-1}=\frac{\Delta x^{2}}{D} \frac{9+M}{2(2+\sqrt{2})+\sum_{k \in \Omega_{i}} \gamma \frac{V_{k}-V_{i}}{d_{i k}^{3} \Delta x}} .
$$


This is a decreasing (increasing) function of $\gamma$ for $\sum_{k \in \Omega_{i}}\left(V_{k}-V_{i}\right) /\left(d_{i k}^{3} \Delta x\right)>$ $0(<0)$ which reflects movement up VEGF gradients. It is also clear that the parameter $M$ measures the tendency of a cell to stay put-as $M$ increases the mean waiting time increases. A similar relationship holds for the mean waiting time for finite $N_{m}$, but we omit it here.

- Diagonal tip cell movement is allowed, except when it would cause one sprout to cross a vessel or another sprout.

- In each lattice site visited by the tip, an endothelial cell is created that remains there without moving (this is similar to the "snail trail" used by other authors [7,47]).

- A tip cell establishes a connection by moving to a lattice site also occupied by a sprout (possibly itself) or by a vessel.

- If a sprout does not connect within a given period of time then the sprout dies and is removed. This rule is based on the fact that ECs depend upon flow-induced signals for survival [42]. As blunt-ended sprouts are not circulated, ECs in unconnected sprouts do not receive these signals. We assume that the maximum time span ECs can survive without these signals is the same as the time, $T_{\text {prune, }}$, that established vessels can survive with low WSS. Note that we consider a single age for the whole sprout, but this could be relaxed in combination with a more detailed model for endothelial cell proliferation behind a tip cell.

- Connected sprouts are re-labelled as ACTIVE and added to the vascular network, with each endothelial cell in a sprout contributing a vessel segment.

- The new vascular network is used to update the blood flow and haematocrit distribution. Since pruning due to low WSS applies to individual vessel segments, single segments in a new angiogenic vessel (or in any path between bifurcating nodes) could be pruned. However, as noted in Sect. 2.1, the rest of any resulting bluntended path will be removed later, unless flow is again established by a subsequent new connection.

We remark that our approach to tip cell movement is similar to the approaches of Anderson et al. [7] and Plank and Sleeman [39], except that we allow diagonal movement. Anderson et al. [7] consider transition probabilities based upon a discretisation of partial differential equations for random motion, chemotaxis and haptotaxis, whereas Plank and Sleeman utilise an approach of Othmer and Stevens [35] with constant mean waiting time. In contrast, our transition probabilities also take account of the space available in neighbouring lattice sites, and give a mean waiting time that depends on VEGF gradients. An alternative is to consider a lattice-free approach $[38,47]$.

\section{Vascular adaptation in healthy tissue}

Here we explore the consequences of vessel pruning and angiogenesis in normal tissue. For clarity, throughout this section we neglect VEGF-induced vessel dilation (as introduced in [4]). We also postpone exploration of the role of asymmetric haematocrit splitting until the discussion, assuming instead that the haematocrit splits symmetrically at bifurcations. 
For all simulations we consider a tissue domain of $50 \times 50$ cellular automaton locations, with a grid spacing $\Delta x=40 \mu \mathrm{m}$. Thus we assume that cells are not particularly closely packed, and the tissue is $2 \mathrm{~mm}$ square. Simulation time is presented in days, with a time step between automata updates of $\Delta t=30 \mathrm{~min}$. The basic parameter set for all simulations is given in Tables 1, 2, 3, 4 in Appendix B.

\subsection{Vessel pruning due to low wall shear stress}

As outlined above, low wall shear stress is thought to be a principal mechanism behind vessel regression $[19,34,42]$. Here we consider a situation where a vascular bed has been established, and we examine the consequences of subsequent pruning. This could mimic a situation of wound healing or vasculogenesis, in which the network forms prior to remodelling. Alternatively, it could represent situations such as occlusion of an upstream feeding vessel, a reduction in blood pressure, or the application of a vasodilator. Figure 2 shows how as the pressure drop across a network is reduced, more vessels are pruned due to low wall shear stress. In each case the initial flow pattern gives rise to higher wall shear stress in paths that lead from the inflow (bottom left) to the outflow (top right), with low levels of wall shear stress in segments that run in the approximately perpendicular direction (i.e. those segments that couple nodes with almost equal pressures). In addition, the most peripheral parts of the network (in terms of distance from the inflow or outflow) also experience the lowest wall shear stress.

\subsection{Angiogenesis}

We begin by considering a situation in which a population of normal cells is initially under-vascularised, with hypoxia in the gap between two parallel parent vessels. Figure 3 shows how initially the normal cells die in the most hypoxic region, whilst those that survive secrete VEGF which stimulates angiogenic sprouting. Many sprouts fail to connect before dying, or experience insufficient wall shear stress for survival, but gradually new functional connections form and are maintained until the simulated tissue becomes well vascularised and VEGF levels fall. At larger times (not shown) VEGF levels fall to zero everywhere and angiogenesis ceases.

We expect that blood oxygenation should affect the vascular density that develops. For lower levels of oxygen release from the blood we would expect to see hypoxia and VEGF levels remain high for longer, and hence more angiogenesis and a higher final vascular density. Figure 4 shows the time evolution, averaged over five stochastic realisations, of vascular density and VEGF for varying oxygen release. In the case of lower oxygen release we see that a higher vascular density develops in order to compensate for the reduced ability of each vessel to supply oxygen.

It is interesting to assess whether we reach a similar endpoint (in terms of average vascular density) if we use different initial vessel configurations. For example, we ran simulations with 1, 2, 3 or 4 equally spaced initial parent vessels, all running from the left to right edge of the domain with the same pressure drop. We found that the long time vascular density is weakly dependent on the initial vascular distribution. With one 

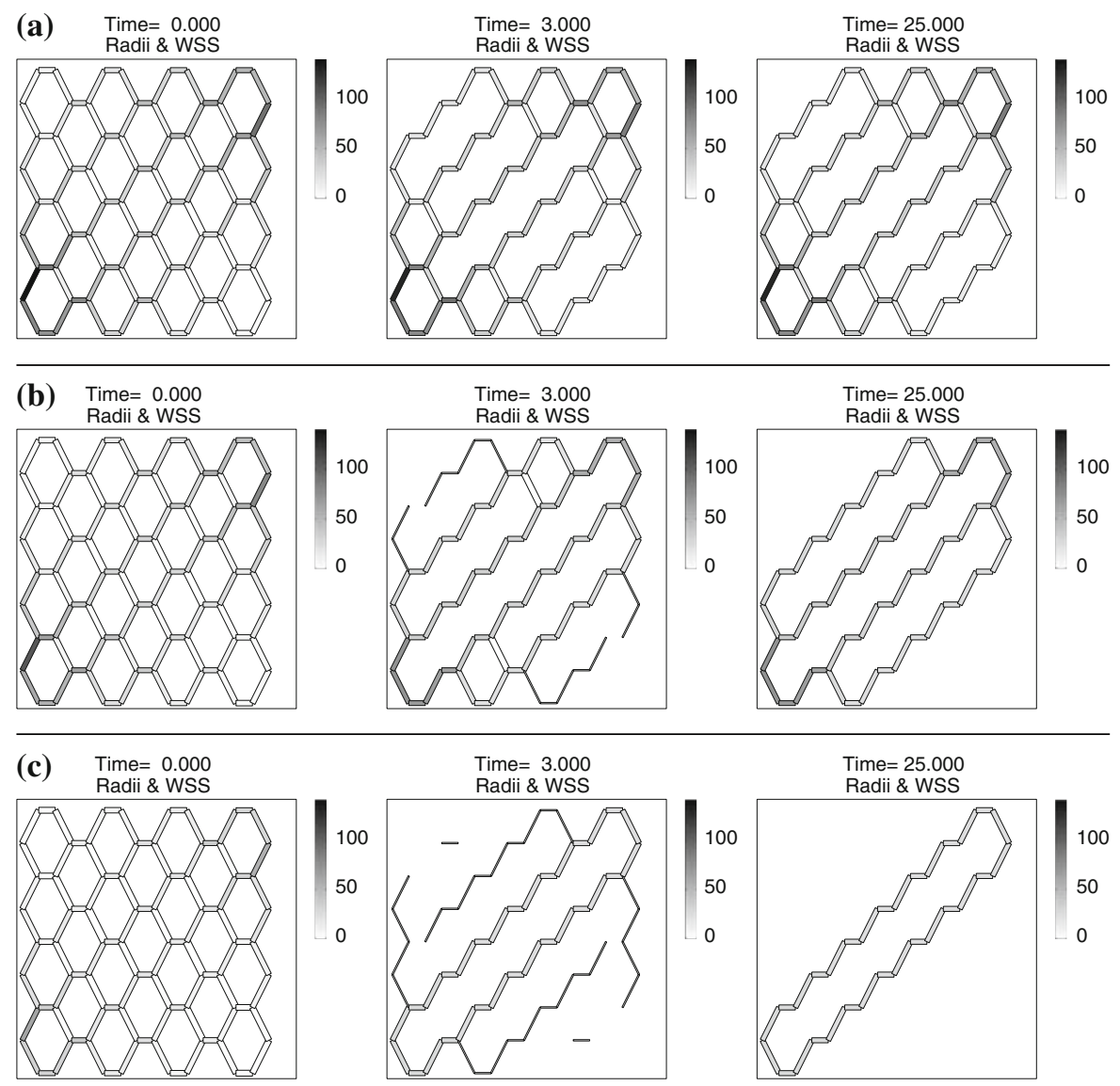

Fig. 2 Pruning of an initially regular hexagonal network, for three values of the pressure difference across the network. This pressure difference is applied from the bottom left node to the top right node. As the pressure difference decreases, the number of vessels in which flow is maintained falls. The inflow pressures were a $P_{\text {in }}=24 \mathrm{mmHg}$, b $P_{\text {in }}=22 \mathrm{mmHg}$, c $P_{\text {in }}=19 \mathrm{mmHg}$, with an outflow pressure in each case of $P_{\text {out }}=15 \mathrm{mmHg}$. The thickness of each segment is proportional to the vessel radius calculated using Eq. (1), and the shading indicates the wall shear stress. Parameter values are as in Tables 1, 2, 3, 4, except for $P_{\text {sprout }}^{\max }=0$ (no angiogenic sprouting) and the inflow pressures indicated above

initial vessel the network is still (weakly) stimulated to develop at time 200, whereas with 2/3/4 initial vessels by time 200 there is little or no hypoxia/VEGF production and hence little or no further sprouting/angiogenesis. Since all initial vessels were linear, and linear vessels oxygenate a greater area of tissue per unit length than more tortuous vessels, we found that with four initial vessels only the top and bottom of the domain see significant angiogenesis, and the tissue reaches a steady vasculature earlier. In contrast, with a single initial vessel by the end of the simulated time a steady state is only just being approached. Thus we see an ordering, with the final vessel density decreasing as the initial number of (linear) parent vessels increases from one to four (results not shown). 


\section{Time $=0.000$}
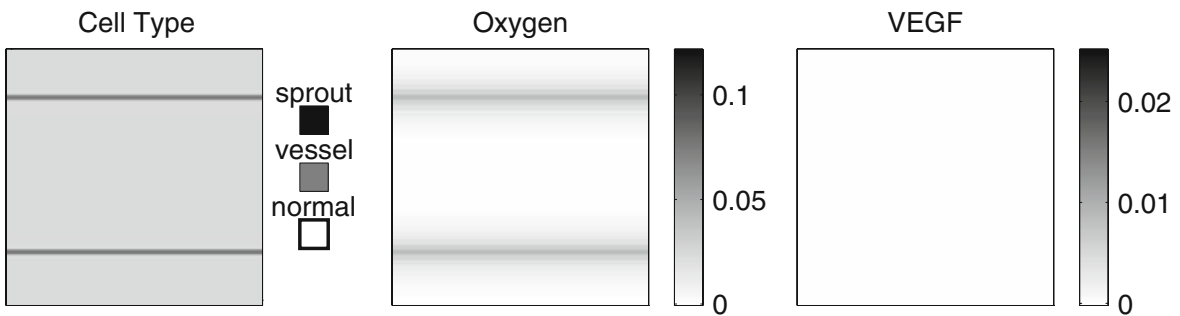

Time $=20.000$
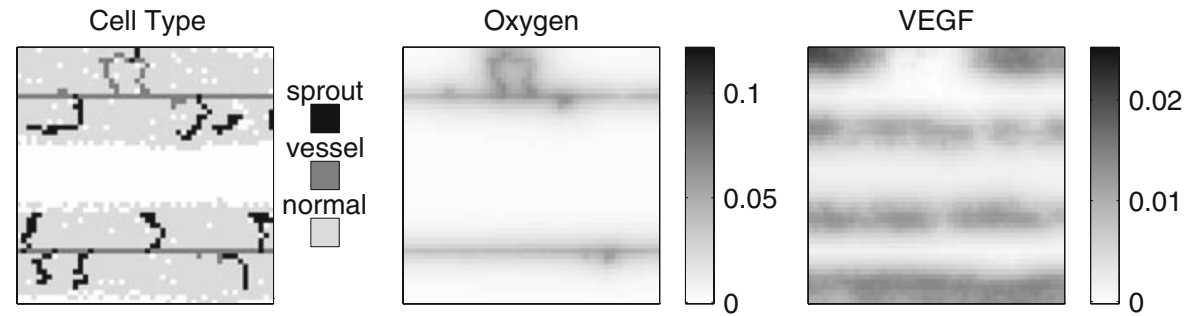

Time $=40.000$
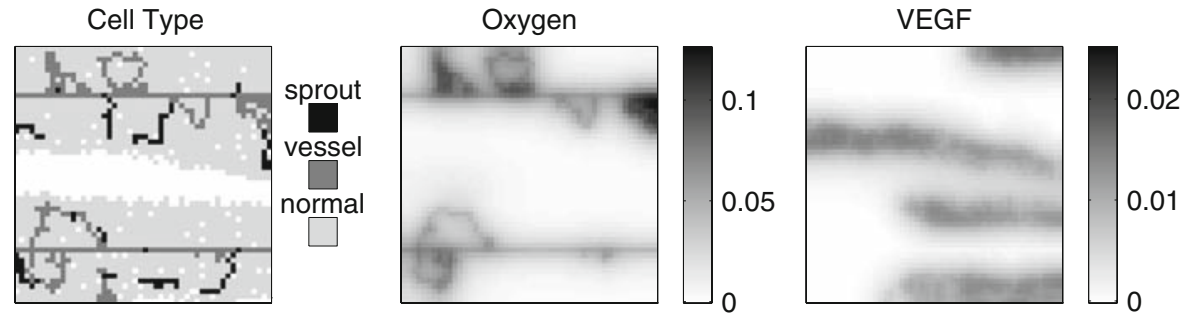

Time $=60.000$
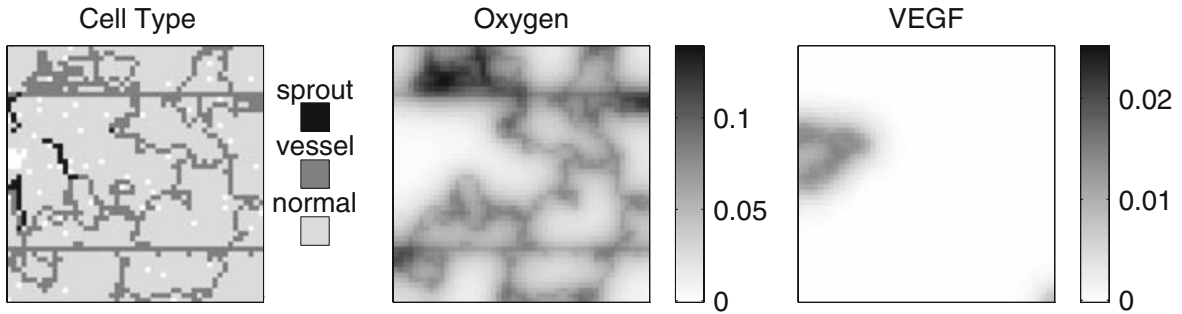

Fig. 3 Evolution, from an initial pair of straight vessels (both with inflow at the left and outflow at the right), of an irregular vascular network via angiogenesis. Note that the normal cells most distant from the original two parent vessels initially die, until angiogenesis provides sufficient oxygen to sustain them. Parameter values are as in Tables 1, 2, 3, 4. A movie of this simulation can be found in the electronic supplementary material

\subsection{Remodelling via pruning and angiogenesis}

We have seen that pruning can eliminate poorly perfused vessels, and that angiogenesis can generate new vessels in response to hypoxia. We now seek to determine whether 

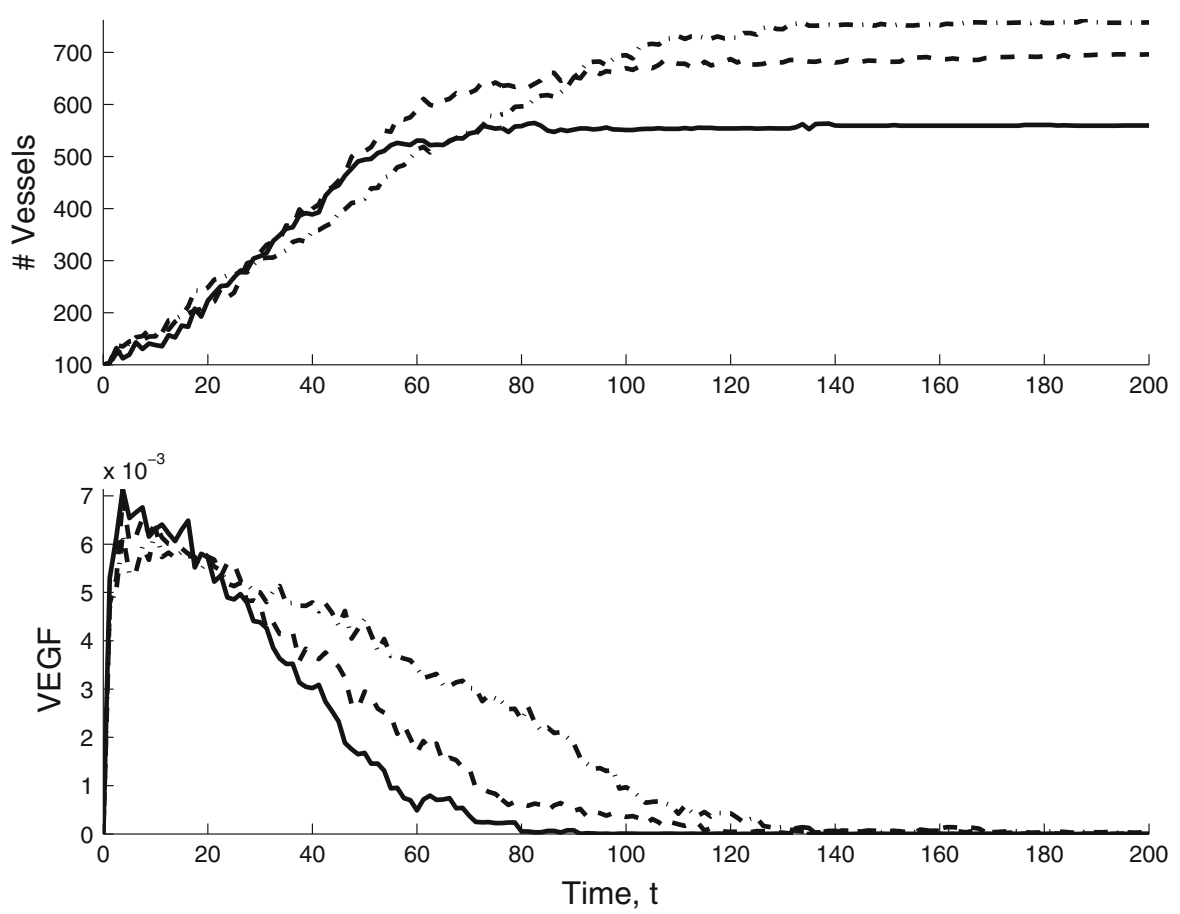

Fig. 4 Summary of the dynamics of vascular density and VEGF for varying oxygen release, averaged over five stochastic realisations. (Solid lines) Oxygen release, $\mathscr{P}_{c}=700$, as in Fig. 3; (dashed lines) intermediate oxygen release, $\mathscr{P}_{c}=500 ;$ (dot-dashed lines) lower oxygen release, $\mathscr{P}_{c}=300$. In each case we show the mean of five simulations. Lower oxygen release means that a higher vascular density is required. Parameter values are as in Tables $1,2,3,4$, except for the different values of $\mathscr{P}_{c}$ indicated above

angiogenesis can restore tissue oxygenation in the cases of vessel pruning outlined in Fig. 2.

Figure 5a shows our first example, using the same inflow pressure, $22 \mathrm{mmHg}$, as in Fig. 2b. Early pruning leaves four main vessel paths from inflow to outflow, and remodelling fails to reoxygenate the whole tissue region. In fact, because the new vessels stimulated by the initial pruning are more tortuous than those that they replace, we actually find a contraction of the area oxygenated. Consequently we considered the case with a chemotaxis coefficient $\gamma$ increased by a factor of ten (Fig. 5b). We now see that remodelling is able to completely supply the tissue region. This process requires the effective replacement of vessels that are not necessary in one region of tissue with new vessels in regions that are undersupplied. Thus, new vessels must form and be maintained by "stealing" sufficient flow from existing vessels. Figure 6 shows the time course of vessel density and mean VEGF concentration, for $\gamma=8 \times 10^{4}$ and $\gamma=8 \times 10^{5}$, averaged over 10 simulations in each case. This illustrates how the vessel density initially falls (due to pruning) before rising to fluctuate about a mean level governed by the pressure drop, while the VEGF level reflects the ability of the evolving vessel network to oxygenate the tissue. 
(a)

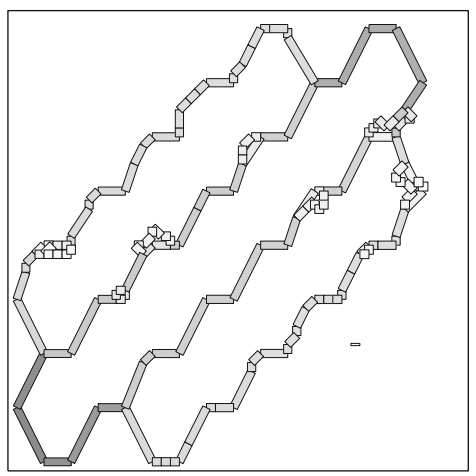

Time $=100.000$

Radii \& WSS

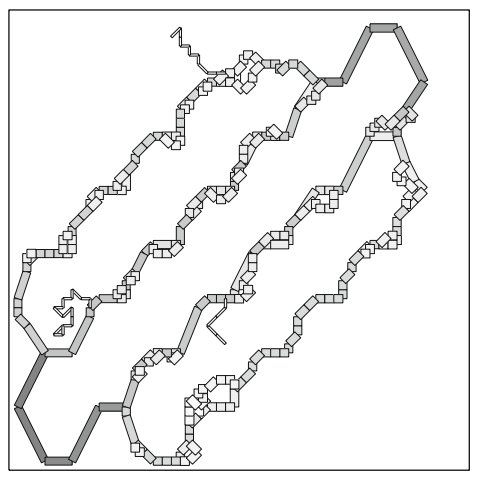

Time $=400.000$

Radii \& WSS

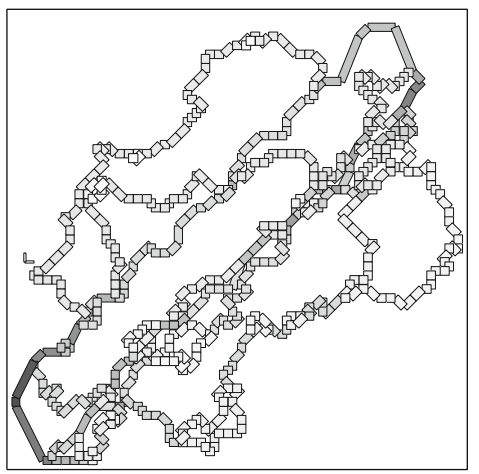

(b) $\begin{array}{r}\text { Time }=25.000 \\ \text { Radii \& WSS }\end{array}$

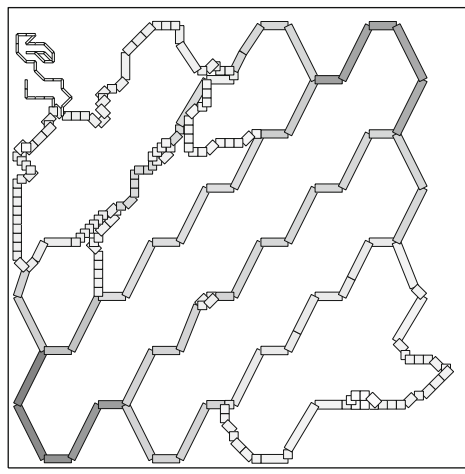

Time $=100.000$

Radii \& WSS

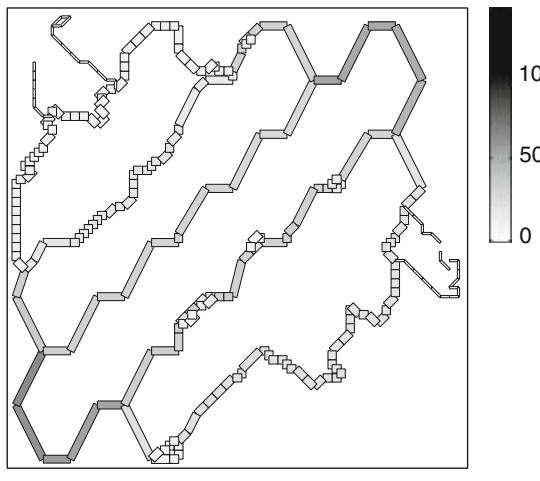

Time $=400.000$

Radii \& WSS

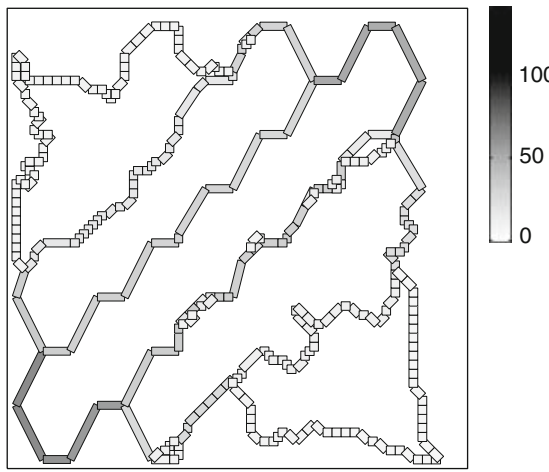

Fig. 5 Pruning followed by angiogenesis. a The chemotactic sensitivity $\gamma=8 \times 10^{4}$, and the new vessels are poorly directed and hence lead to poorer vascularisation. b Increasing the chemotaxis coefficient to $\gamma=8 \times 10^{5}$ gives more rapid and better directed sprout growth, hence effectively remodelling the vasculature and oxygenating the whole tissue region. Parameter values are as in Tables 1, 2, 3, 4, except for $P_{i n}=22 \mathrm{mmHg}$, and the different value of $\gamma$ in (b). Movies of these simulations can be found in the electronic supplementary material 

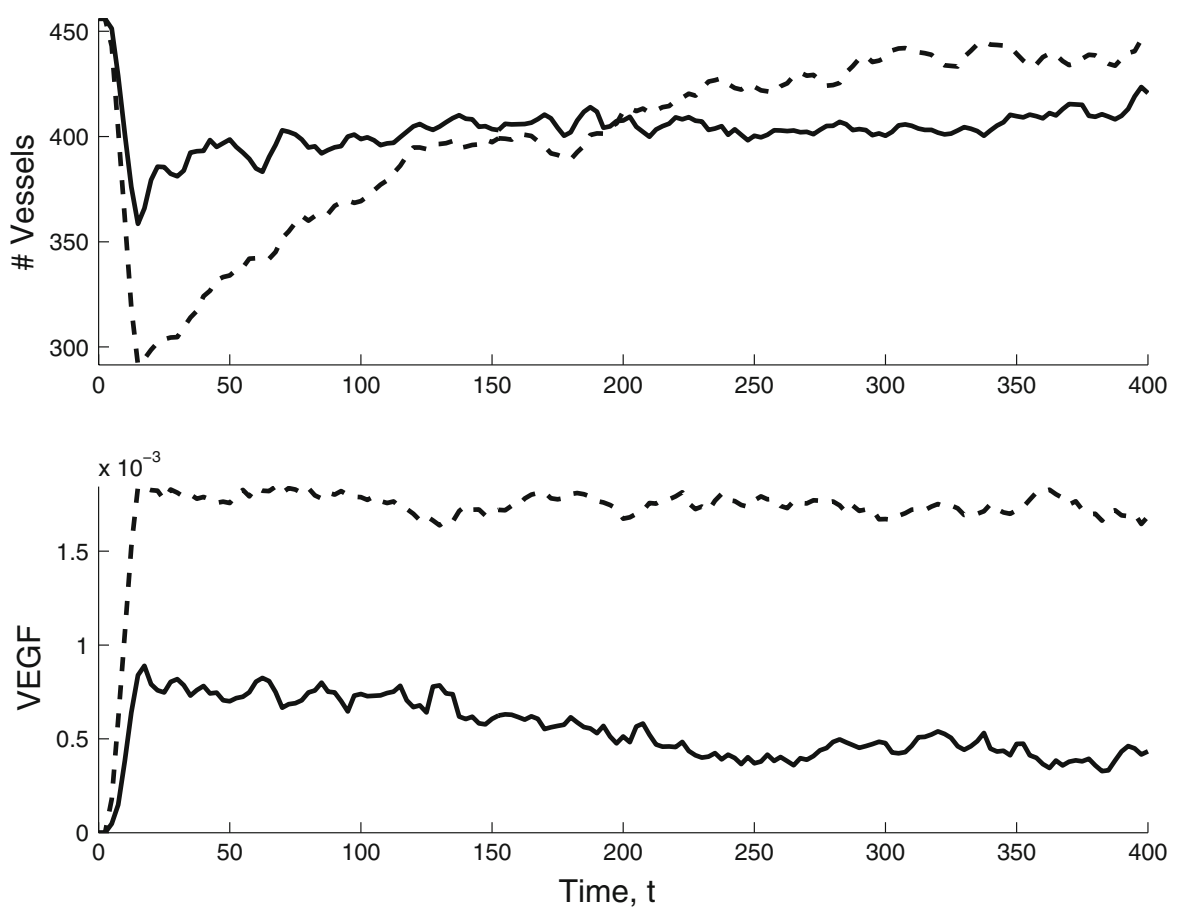

Fig. 6 Summary of the mean dynamics of vessel number and VEGF levels for ten simulations with the parameter sets depicted in Fig. 5a (dashed lines $\gamma=8 \times 10^{4}$ ) and Fig. $5 \mathrm{~b}$ (solid lines $\gamma=8 \times 10^{5}$ ). For $\gamma=8 \times 10^{4}$ the mean number of vessels fluctuates around a level sustainable by the pressure difference across the vessel network, whilst the mean VEGF concentration remains high. For $\gamma=8 \times 10^{5}$ remodelling is able to oxygenate more of the tissue, so that, on average, VEGF levels are much smaller. We show a moving time-average of each quantity for clarity (a window size of 10 time units is used). Parameter values are as in Tables $1,2,3,4$, except for $P_{\text {in }}=22 \mathrm{mmHg}$, and the case with $\gamma=8 \times 10^{5}$

We also check that this process can vascularise the same tissue region from a single initial vessel. Figure 7 a shows the initial condition, and Fig. 7b shows a simulation corresponding to the case of Fig. 5b. Early in the simulation, normal cells far from the initial parent vessel die due to hypoxia-induced apoptosis, but VEGF production stimulates angiogenic sprouting and the whole region is repopulated in a wave-like manner as new vessels connect, allowing normal cells to proliferate and spread, which in turn triggers further angiogenesis. Figure $7 \mathrm{c}$ shows an example where a lower inflow pressure alters this balance in favour of vessel regression. As a result, the vessel network cannot extend across the whole region, normal cells cannot survive in the hypoxic corners, and hypoxia induced VEGF production is not eliminated. Figure 8 shows the time course, averaged over ten realisations in each case, for these two different inflow pressures. For the larger pressure drop, VEGF levels initially rise sharply, before declining as vascularisation progresses (which is marked by the number of vessels rising and then levelling off). For the lower pressure drop, the vessel number approaches a lower level, whilst VEGF levels remain high, reflecting the dominance of vessel pruning and consequent sustained hypoxia. 
(a) Cell Type

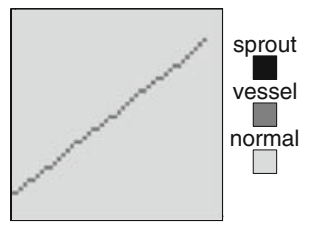

(b) Cell Type

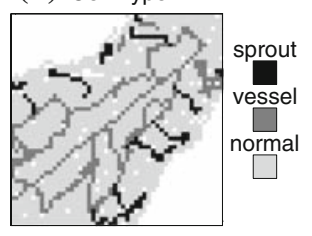

Time $=0.000$

Radii \& WSS

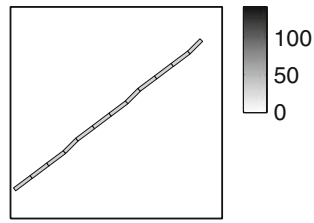

Time $=25.000$

Radii \& WSS
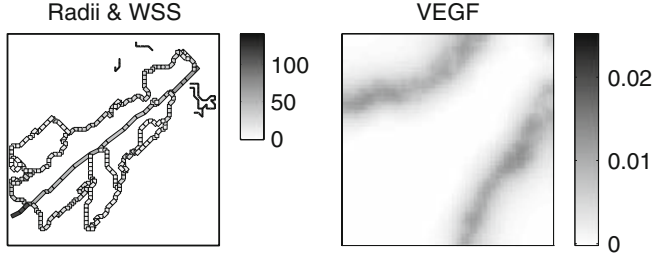

Time $=800.000$

Radii \& WSS
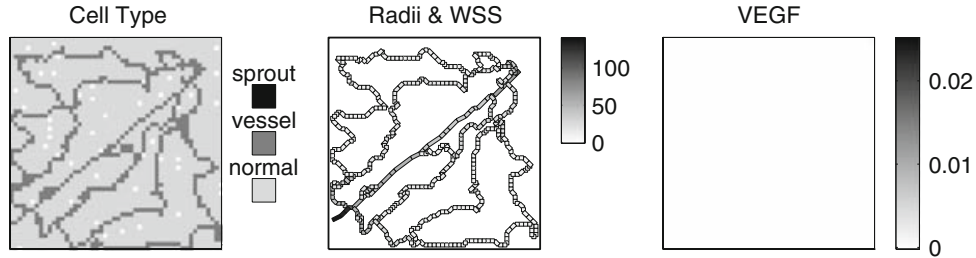

0.02

(c) Cell Type

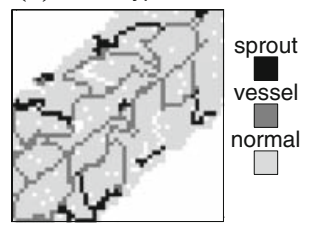

$$
\text { Radii \& WSS }
$$
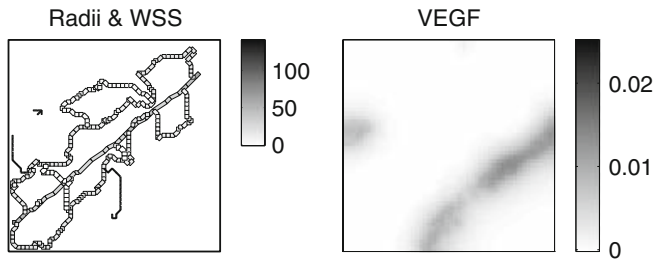

Time $=800.000$
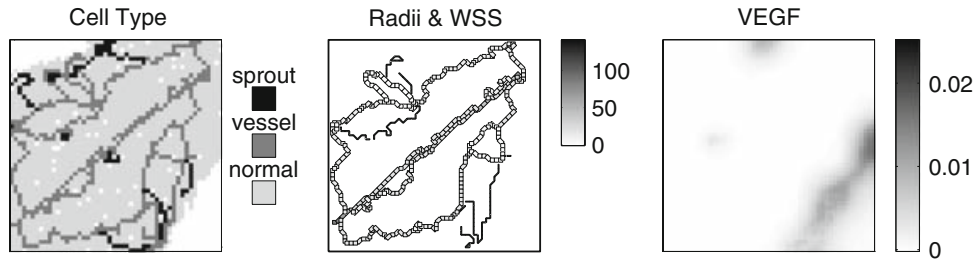

Fig. 7 Angiogenesis from a single initial vessel. a Initial condition. b Parameters as for Fig. 5b, including an inflow pressure $P_{\text {in }}=22 \mathrm{mmHg}$. From a single initial vessel the whole tissue region is fully oxygenated after 800 time units. At first, normal cells far from the parent vessel die. Then the whole region is repopulated in a wave-like manner as new vessels and normal cells grow together. $\mathrm{c}$ For a lower inflow pressure, $P_{\text {in }}=19$ $\mathrm{mmHg}$, the balance between angiogenesis and vessel regression is altered, so that the vessel network cannot extend across the whole region, and hypoxia induced VEGF production is not eliminated. Movies of these simulations can be found in the electronic supplementary material 

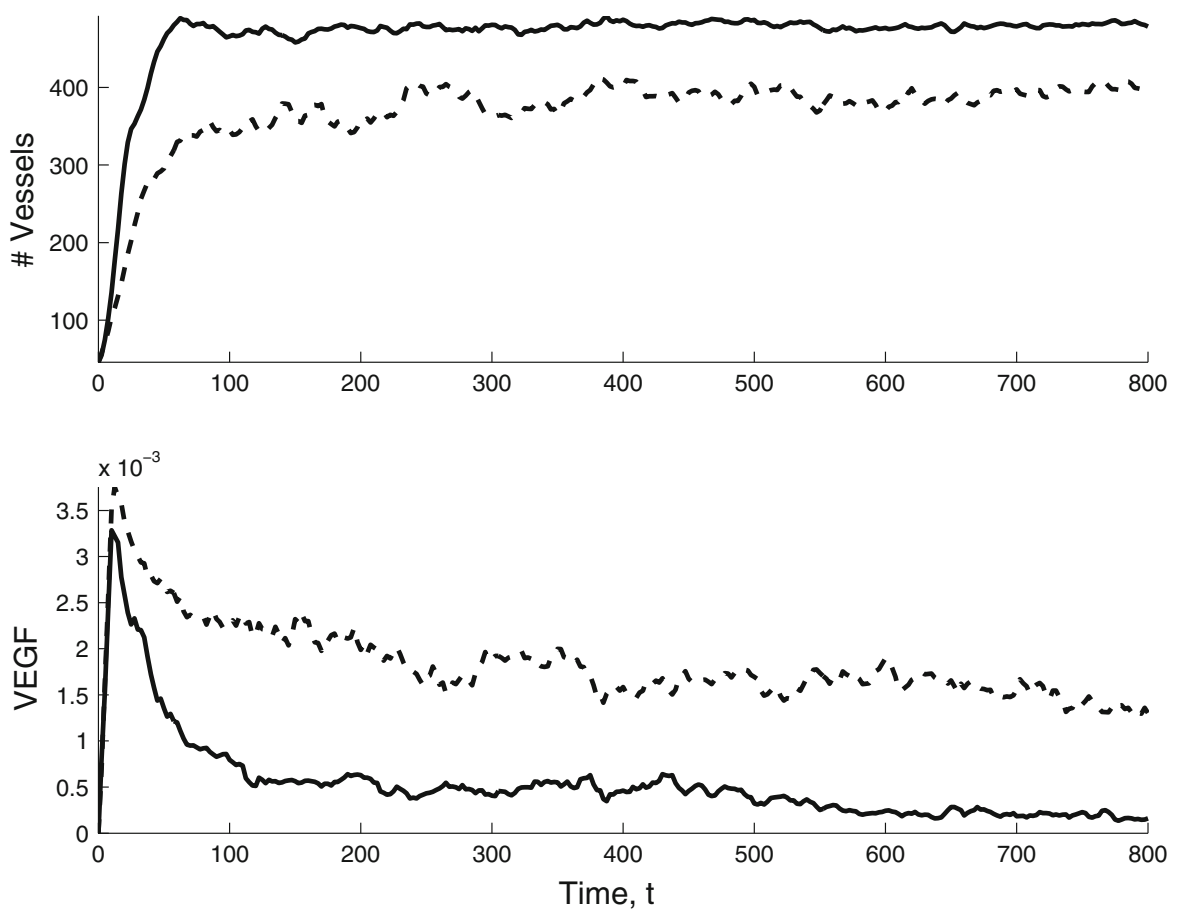

Fig. 8 Summary of the dynamics of angiogenesis from a single initial vessel, as illustrated in Fig. 7. We show the average, over ten simulations, of the vessel number and VEGF levels for varying inflow pressures: (solid line) $P_{\mathrm{in}}=22 \mathrm{mmHg}$, (dashed line) $P_{\mathrm{in}}=19 \mathrm{mmHg}$, with an outflow pressure in each case of 15 $\mathrm{mmHg}$. If the inflow pressure is too low, angiogenesis fails to oxygenate the whole tissue region, and VEGF levels remain high. As in Fig. 6 we show a moving average for clarity (with a window size of 10 time units)

\section{Vascular tumour growth}

We first compare simulations of tumour growth in the absence of angiogenesis, with VEGF-induced vessel dilation as outlined previously [4], and with explicit angiogenesis as introduced here. Figure 9a shows an initial condition with a clone of tumour cells implanted in a tissue with an initially hexagonal vascular network. The cancer cells invade the normal cell population since they have a proliferative advantage, consume more oxygen, and can lower the normal cells' p53 threshold for apoptosis (see Eqs. (16) and (17) in Appendix A.2). Figure 9b shows the long term behaviour with no vessel dilation or angiogenesis, and Fig. 9c shows the case with implicit angiogenesis via VEGF-induced vessel dilation. In each case hypoxic and necrotic regions develop at the centre of each hexagon, with vessel dilation simply making these regions smaller, since each dilated vessel supplies more oxygen to the tissue. With explicit angiogenesis (Fig. 9d), the VEGF produced by quiescent tumour cells leads to angiogenic sprouting and the formation of new functional vessels, and the tumour is able to spread throughout the domain.

We now embed a tumour in normal tissue with two linear vessels. Figure 10 shows that, given this relatively sparse initial vessel network, the tumour remains confined 
(a) Cell Type

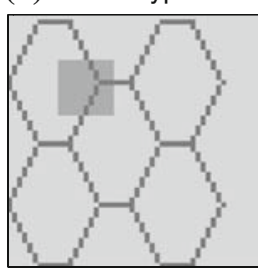

Time $=0.000$

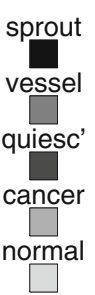

Radii \& WSS

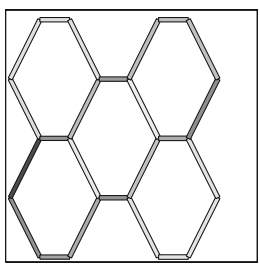

VEGF

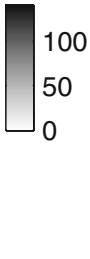

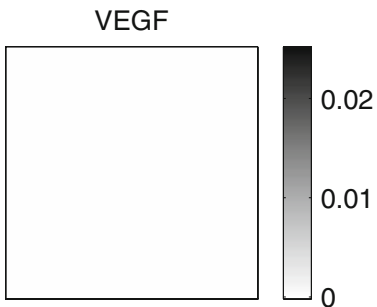

Time $=40.000$

(b) Cell Type

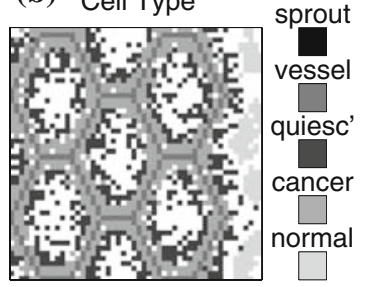

Radii \& WSS

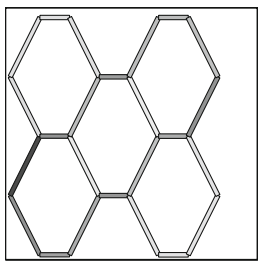

VEGF

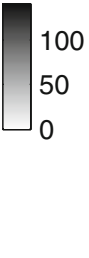

Time $=40.000$

(c) Cell Type

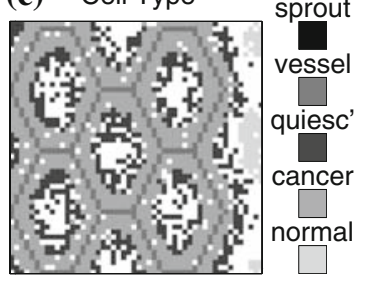

Radii \& WSS

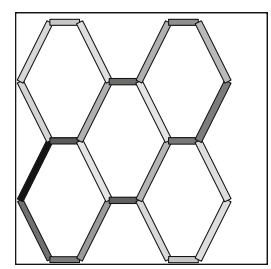

VEGF

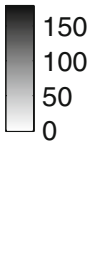

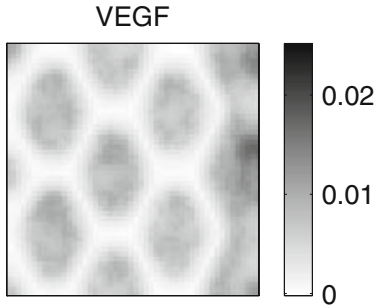

Time $=40.000$

(d) Cell Type

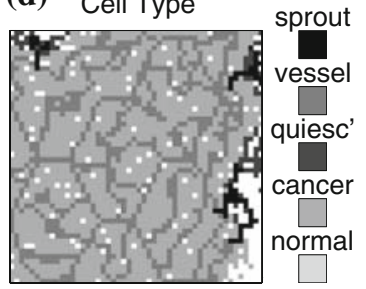

Radii \& WSS

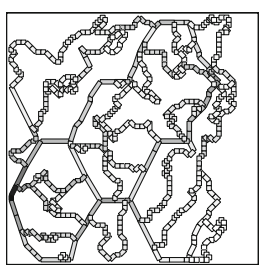

VEGF

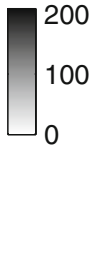

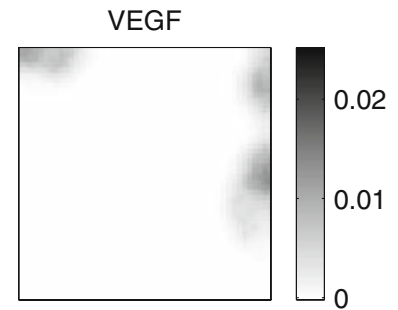

Fig. 9 a Initial condition: a tumour is placed in a hexagonal vessel network. b Long term behaviour with no VEGF-induced vessel dilation or angiogenesis $\left(k_{m}^{V}=P_{\text {sprout }}^{\max }=0\right)$. The tumour rapidly spreads wherever there are vessels, but hypoxic and necrotic regions arise elsewhere, leading to high VEGF levels. $\mathbf{c}$ With VEGF induced vessel dilation but no angiogenic sprouting $\left(k_{m}^{V}=10, P_{\text {sprout }}^{\max }=0\right)$ the hypoxic/necrotic regions shrink due to enhanced oxygen delivery. $\mathbf{d}$ With angiogenesis but no vessel dilation the tumour is able to spread throughout the domain, although regions of hypoxia/necrosis come and go depending on the balance between angiogenesis and flow (low flow can cause vessel elimination). Parameter values are as in Tables 1, 2, 3, 4, except as indicated above 
close to a single parent vessel (analogous to tumour cords [13]) until new vessels bridge the gap to another nearby parent vessel. This can lead to periods of relative stagnation followed by sudden expansions in tumour size (the number of tumour cells rises steeply around $t=30$ ). In contrast, if we first allow the normal vessel network to develop via angiogenesis (and hence use the final network from the simulation of Fig. 3 as our new initial condition), the tumour spreads more rapidly, exploiting the relatively dense initial vasculature and adding to it where hypoxia arises. Figure 11 shows an example simulation, and Fig. 12 shows the time courses (averaged over five realisations) of the numbers of tumour cells and vessels. Although the initial tumour growth is more rapid, the long-term tumour size and vascular density are the same (on average) with and without a dense initial vascular network. In addition, the long-term vascular density with a tumour is significantly higher than that without a tumour, due to the cancer cells' higher oxygen consumption rate.

\section{Discussion}

We have incorporated angiogenesis and vascular remodelling within a previously developed multiscale model for vascular tissue growth $[2,4,5,14]$. We include two fundamental mechanisms of vascular development and homeostasis, (1) by explicitly accounting for the pruning of vessels that have insufficient flow for a sustained period of time, and (2) via VEGF-dependent formation of angiogenic sprouts and the consequent creation of new vessel connections that establish blood flow. These two mechanisms provide the ability to generate new vasculature to supply regions with too low a vascular density, and to eliminate vessels that do not sustain flow.

We found that vessel pruning is principally determined by the pressure drop across the vascular network, with a smaller drop leading to more pruning (see Fig. 2). This is similar to the emergence of a dilated backbone in the work of McDougall and co-workers [33], except that here even large vessels can be pruned if their wall shear stress falls. With angiogenesis, the level of blood oxygenation regulates the extent of angiogenesis and the mean long term vascular density, with higher oxygenation leading to fewer vessels. We also found that the initial vasculature can affect the final vascular density, particularly when the pressure drop across the network is high enough to support low levels of vessel pruning. Combining these insights, simulations show that network remodelling is best achieved via an appropriate balance between pruning and angiogenesis. It is also important that the chemotactic bias in sprout migration is sufficiently strong to form vessels that are not too tortuous (Figs. 5, 6). The fluid flow calculations do not explicitly include any notion of tortuosity, but given two connections, with the same start and end points, the same radii and an equal pressure drop, the longer connection (and hence typically more tortuous) will have the lower wall shear stress and hence is more likely to be pruned (this argument does not take into account the effect of curvature on wall shear stress). This dependence on the strength of chemotaxis raises an interesting question as to whether the trend persists, or whether a sufficiently strong chemotactic bias could in fact be detrimental (for example by overextending new vessels, which could again give lower wall shear stress). 
Time $=0.000$
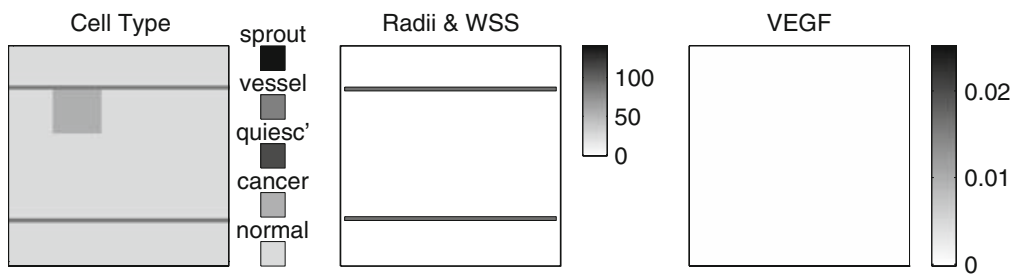

Time $=20.000$

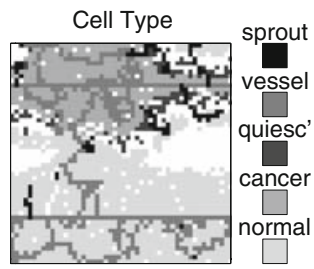

Radii \& WSS
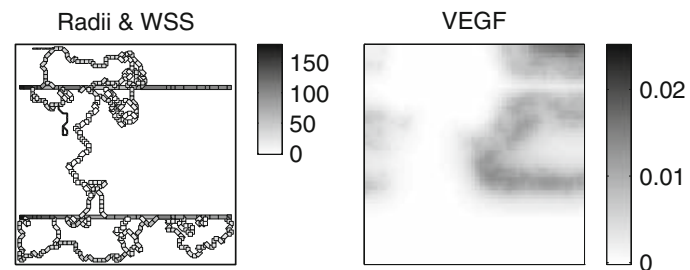

Time $=40.000$

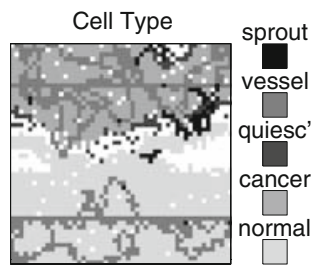

Radii \& WSS
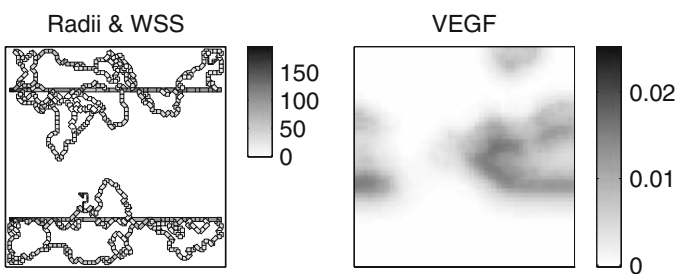

Time $=60.000$

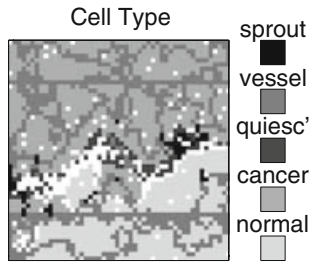

Radii \& WSS
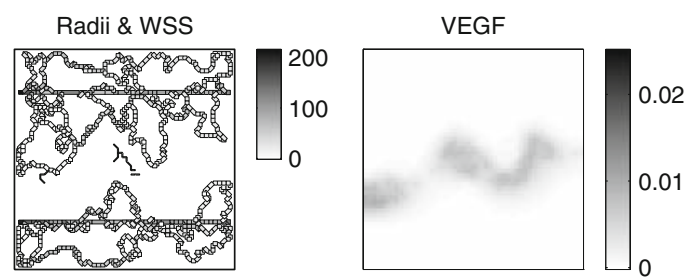

Time $=80.000$

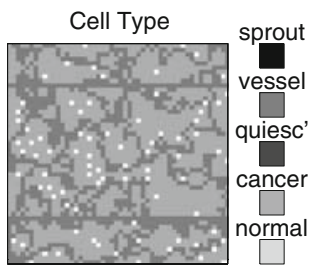

Radii \& WSS
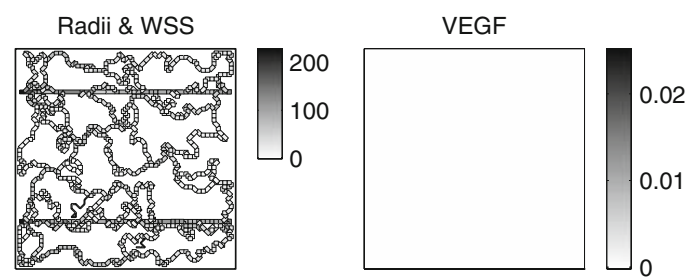

Fig. 10 A simulation with tumour cells implanted in a tissue with normal cells and two linear initial vessels (as in Fig. 3). Notice how the tumour remains confined close to the upper vessel until connections are made that allow it to spread fully into the lower half of the domain. Also worth noting is that tumour cells' increased oxygen consumption triggers VEGF expression by more normal cells as well, since they also experience resultant low oxygen levels. The final vascular density is significantly higher than with normal cells only (see Fig. 12). Parameter values are as in Tables 1, 2, 3, 4. A movie of this simulation can be found in the electronic supplementary material 
Time $=0.000$

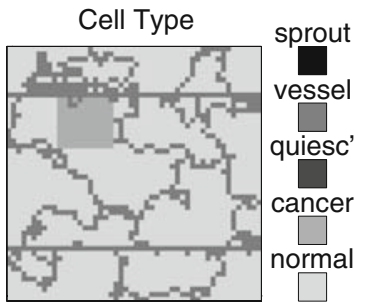

Radii \& WSS
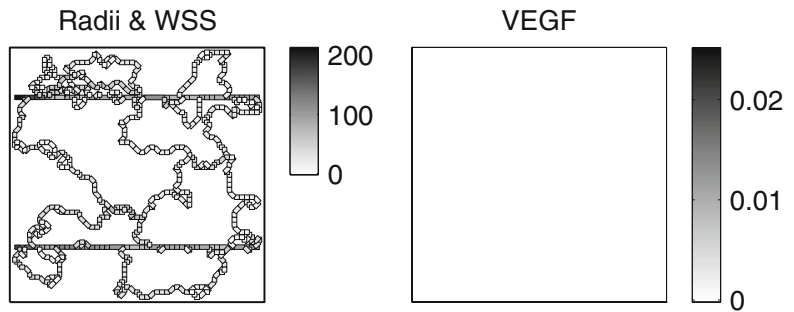

Time $=10.000$

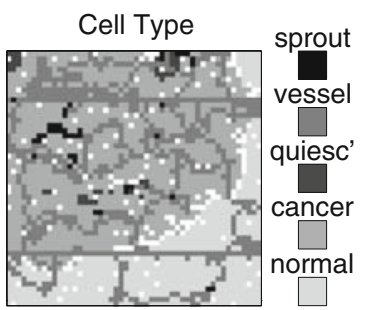

Radii \& WSS
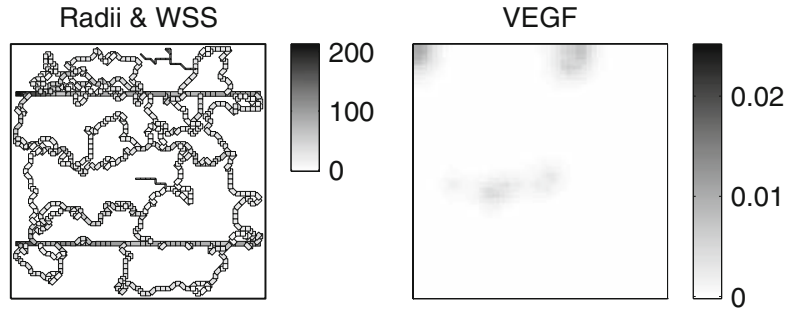

Time $=20.000$

Cell Type

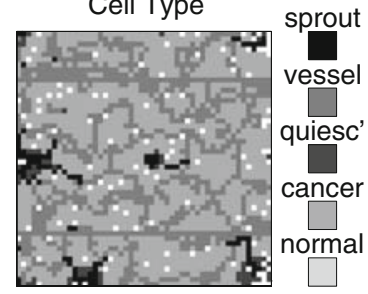

Cell Type

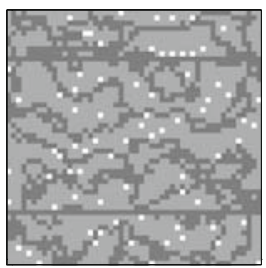

Radii \& WSS
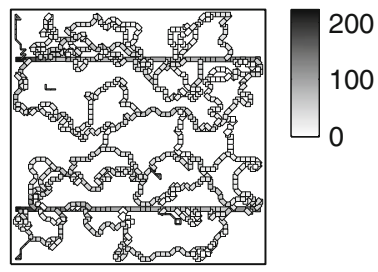

Time $=200.000$
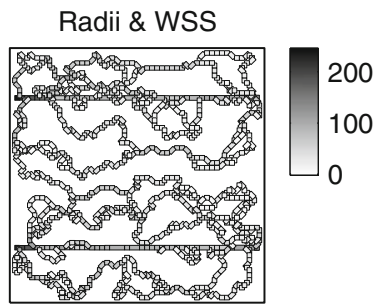
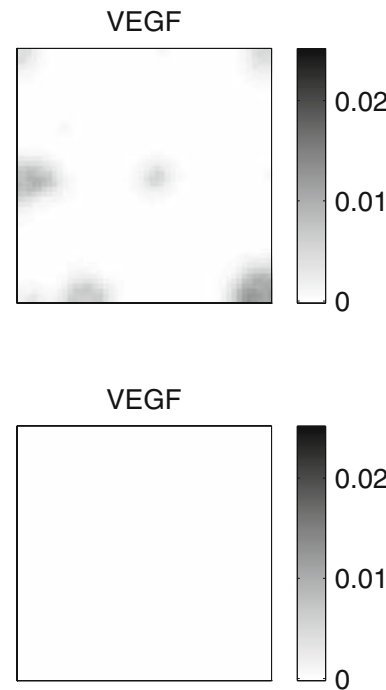

Fig. 11 Evolution following the implantation of a cluster of tumour cells into a previously developed vessel network. The initial vasculature is taken from the end (i.e. at time $=200$ ) of the normal tissue simulation of Fig. 3. Compared to Fig. 10, the tumour spreads more rapidly due to the denser initial vasculature. Further angiogenesis and remodelling proceeds more slowly, until the whole domain is sufficiently well oxygenated. Parameter values are as in Tables 1, 2, 3, 4

When a cluster of tumour cells is introduced into normal tissue, tumour growth is accompanied by the formation of hypoxic regions and the production of VEGF by quiescent cells, leading to angiogenic sprouting, and the vascular density rises above levels for normal tissue alone. We also found that if the original vessel network is 

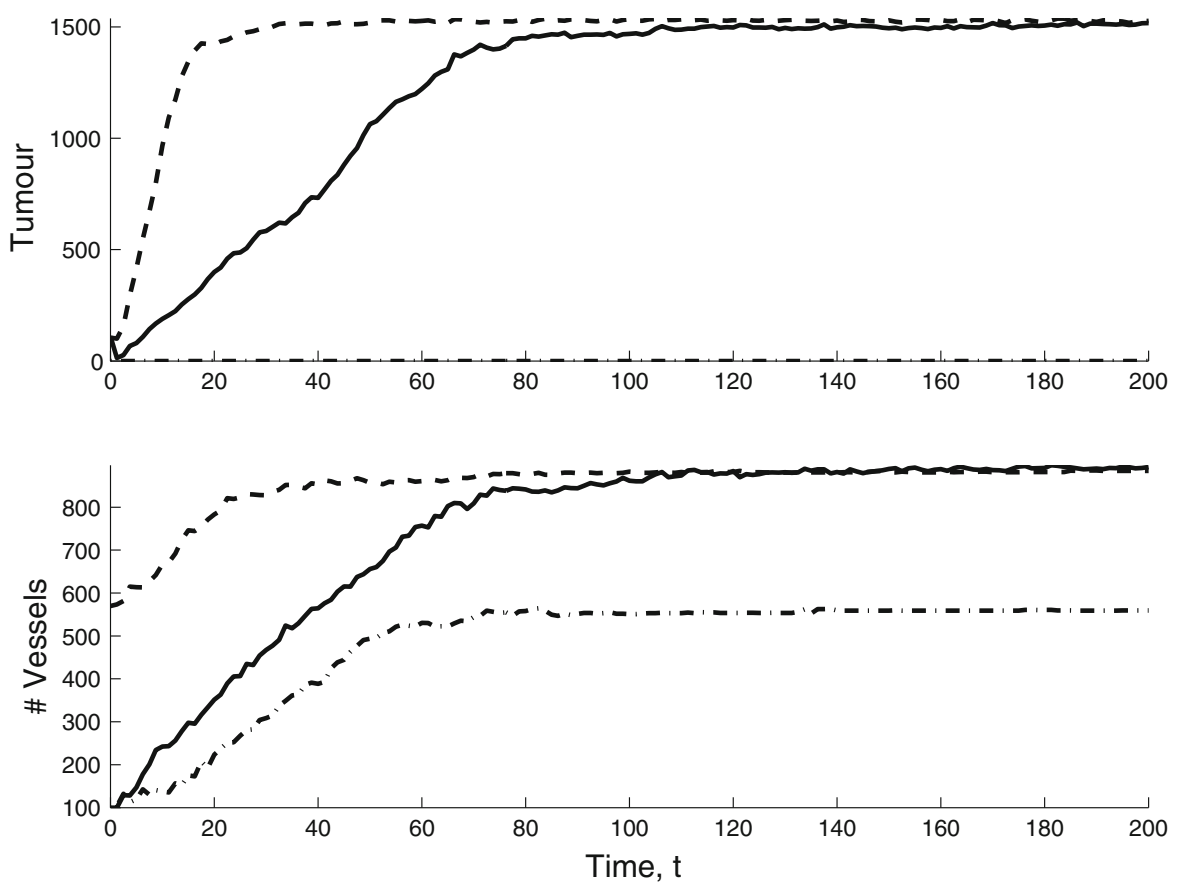

Fig. 12 Time evolution of the number of tumour cells and vascular density. (Solid) Tumour cells added to an initial vasculature of two linear vessels, as in Fig. 10. (Dashed) Tumour cells added to the final vasculature from the normal simulation of Fig. 3 (see Fig. 11). The more dense initial vascular structure means that the tumour can spread far more rapidly, but the long-term vascular density evolves to a similar level. (Dash-dot) For reference we show the evolution of the number of vessels in the absence of tumour cells (the same as the solid curve of Fig. 4). This illustrates how the greater nutrient consumption of tumour cells stimulates a higher vascular density. Each curve is the mean of five simulations. Parameter values are as in Tables 1, 2, 3, 4

sufficiently sparse, the tumour can remain confined close to a single parent vessel (analogous to tumour cords [13]) until new vessels bridge the gap to another nearby parent vessel. This can lead to periods of relative stagnation followed by sudden expansions in tumour size (Fig. 10). Bartha and Rieger [11] concluded that vascular density might not be an appropriate indicator of tumour prognosis, as is generally believed. Instead, their model suggests that tumour progression, at least in its early stages, is strongly determined by the vascular density of the original host tissue. This is supported by our simulations of tumour growth, which also indicate that host tissues of low vascular density give rise to tumour expansion which is limited by the time taken to co-opt the host vasculature.

Up to now we have concentrated on the case without asymmetric haematocrit splitting at bifurcations in order to focus on the dynamics of vessel pruning and angiogenesis. We expect that haematocrit splitting will alter the details in any particular case but that it will not significantly alter the qualitative and time-averaged behaviour. When haematocrit splitting according to Yen and Fung [54] is included, we see that the overall dynamics is very similar (results not shown). Inhomogeneous haematocrit 
distributions may affect local flow patterns, leading to a variation in precisely which vessels are pruned due to low wall shear stress. We found that the vessel pruning simulations of Fig. 2 (where the inflow pressure was varied) showed no difference in the vessels pruned or the final vessel structure. VEGF induced vessel dilation was found to be a stronger factor, leading to the deletion of extra vessels, because dilated vessels typically have lower wall shear stress. As another example we revisited the simulations from Sect. 3.2. We found that asymmetric haematocrit splitting makes little qualitative difference to the vasculature that develops, and that the dynamics and vessel densities reached are very similar with or without asymmetric haematocrit splitting (results not shown).

The multiscale model developed here has diverse applications including to normal vascular development, tumour growth, and wound healing. There are almost innumerable possible directions for future work, ranging from the inclusion of more detail at the subcellular level to the incorporation of additional cell types such as macrophages. Extravasation of other cell types is a straightforward modification of the rules for endothelial tip cell sprouting, so we can now use our model to study issues such as drug delivery using engineered macrophages [36] (which can migrate chemotactically using the formalism of Eq. 8), invasion [6], or even intravasation of tumour cells and metastasis. We have not so far discussed interactions with the extracellular matrix (ECM), although this is an important ingredient in the angiogenesis models of Anderson and coworkers [7,18], which include haptotaxis, the directed movement of cells in response to gradients in ECM density. In the context of the model described here, it seems likely that including ECM degradation and haptotaxis may promote the formation of less tortuous vessels by reinforcing the direction of tip cell movement.

Other important features to include will be the explicit proliferation of endothelial cells behind the moving tip cell, and modelling of tip cell selection based on the lateral-inhibition process outlined in [24,44,48]. Interestingly, Bentley et al. [12] have recently developed an agent-based computational model for Notch-mediated tip cell selection. Partial differential equation modelling has also been used to address the initiation of angiogenic sprouting, although not specifically tip cell selection $[29,30]$. The angiopoietins also represent an important class of signals involved in vessel maturation and competence for angiogenesis. Further avenues for future investigation include the impact of therapy on vascular tumour growth using different drugs and different protocols, particularly to compare the impact of anti-vascular drugs that target functional vasculature (causing vessel collapse) with those that target proliferating endothelial cells.

Acknowledgments MRO acknowledges the support of the BBSRC (E18413) and thanks the EPRSC for financial support for the Interdisciplinary Angiogenesis Network (ANGIONET, EP/D501083/1, http://www.maths.nottingham.ac.uk/angionet/). TA thanks the EPSRC for financial support. PKM acknowledges the support of NIH Grant U56CA113004 from the National Cancer Institute and a Royal Society-Wolfson Research Merit Award. HMB thanks the EPSRC for funding as an Advanced Research Fellow. Part of this work was conducted during the Special semester on Quantitative Biology Analyzed by Mathematical Methods, 1 October to 27 January 2008, organised by RICAM, Austrian Academy of Sciences. 


\section{Appendix A: Mathematical details of the multiscale model}

The overall structure of the multiscale model simulation is as follows:

1. Initialise the vascular network, cell distributions, states of the subcellular ODEs, and distributions of oxygen and VEGF.

2. Update the cells, oxygen and VEGF.

- UpdateOxygen: finite differences applied to Eq. (5).

- Integrate cell cycle and p53-VEGF ODEs (see A.1) over $\Delta t$.

- CellDivision: see A.2

- CellMovement: transition probabilities according to Eq. (8).

- UpdateVEGF: finite differences applied to Eq. (6).

- CellQuiescence: see A.2

- CellDeath: see A.2

3. Update the vasculature.

(a) TipCellSelection: new tip cells with probability given by (7), see Sect. 2.2.

(b) Anastomosis: see Sect. 2.2.

(c) Pruning: Check all vessels and prune if $\tau_{w}<\tau_{w}^{\text {crit }}$ for longer than $T_{\text {prune }}$.

(d) Calculate flows, haematocrit and radii, iterate until $\left(|\Delta R / R|<R_{\text {reltol }}\right)$. See Sect. A.3.

4. Repeat steps $2 \& 3$.

In the following sections we briefly summarise the details indicated in 2 and 3 above.

\section{A.1 Subcellular}

Here we summarise the submodels for the cell cycle and p53-VEGF. The reader is referred to reference [4] for a fully detailed account. The cell cycle model, introduced in [3] as an extension of the Tyson-Novak framework [51], accounts for the effects of hypoxia via the modulation of p27 degradation. The model consists of five ordinary differential equations for the cell mass and the proteins Cdh1, cycCDK, p27 and npRB.

$$
\begin{aligned}
\frac{d[C d h 1]}{d t} & =\frac{\left(1+b_{3}[n p R B]\right)(1-[C d h 1])}{J_{3}+1-[C d h 1]}-\frac{b_{4} M[c y c C D K][C d h 1]}{J_{4}+[C d h 1]} \\
\frac{d[c y c C D K]}{d t} & =a_{4}-\left(a_{1}+a_{2}[C d h 1]+a_{3}[p 27]\right)[c y c C D K] \\
\frac{d M}{d t} & =\eta M\left(1-\frac{M}{M^{*}}\right) \\
\frac{d[p 27]}{d t} & =c_{1}\left(1-\chi \frac{M}{M^{*}}\right)-\frac{c_{2} C(\mathbf{x})}{B+C(\mathbf{x})}[p 27] \\
\frac{d[n p R B]}{d t} & =d_{2}-\left(d_{2}+d_{1}[c y c C D K]\right)[n p R B]
\end{aligned}
$$


The parameters are given in Table 1, except for those that differ between normal and cancer cells, which can be found in Table 2. For example, normal cells produce p27 at rate modulated by their mass, whereas cancer cells are assumed to produce p27 at a constant rate. This is reflected in the values of $\chi$ in Table 2. Also notice that the rate of p27 degradation, and hence the speed of the cell cycle, is an increasing function of oxygen, $C(\mathbf{x})[3]$.

The evolution of the concentrations of p53 and VEGF within a given cell are modulated by oxygen in the following manner:

$$
\begin{aligned}
\frac{d[p 53]}{d t} & =k_{7}-k_{7}^{\prime} \frac{C(\mathbf{x})}{C_{p 53}+C(\mathbf{x})}[p 53] \\
\frac{d[\mathrm{VEGF}]}{d t} & =k_{8}+k_{8}^{\prime \prime} \frac{[p 53][\mathrm{VEGF}]}{J_{5}+[\mathrm{VEGF}]}-k_{8}^{\prime} \frac{C(\mathbf{x})}{C_{\mathrm{VEGF}}+C(\mathbf{x})}[\mathrm{VEGF}] .
\end{aligned}
$$

Here, $k_{8}^{\prime \prime}$ is positive for tumour cells (p53 increases VEGF production in cancer cells), but negative in normal cells (p53 decreases VEGF production in normal cells). Oxygen promotes p53 and VEGF degradation, and their expression therefore increases under hypoxia. For details of the experimental evidence supporting these forms we refer the reader to [4].

Initial conditions for the above differential equations, which are set when a cell is first created, are given by the relevant variable with a subscript zero in Table 1.

\section{A.2 Cellular level}

Cells attempt to divide when $[C d h 1]<C d h 1_{\mathrm{THR}}$ and $[c y c C D K]>\operatorname{cyc} C D K_{\mathrm{THR}}$. A daughter cell is placed in the current location $i$ if the number of cells there is less that the carrying capacity for division (i.e. if $N_{i}<D_{m}$ ). Otherwise, a daughter cell is placed in the neighbouring empty location with the highest oxygen concentration. If neither of these is possible, the parent cell dies. After successful division the parent cell mass is halved and its p27 level is reset to $[p 27]_{0}$, and the daughter cell is initialised as above.

Normal cell apoptosis occurs when $[p 53]>p 53_{\mathrm{THR}}$, where the threshold depends on the local ratio of normal cells to normal and cancer cells, $\rho_{\text {normal }}$ :

$$
p 53_{\mathrm{THR}}=\left\{\begin{array}{ll}
p 53_{\mathrm{THR}}^{\mathrm{high}} \text { for } \rho_{\text {normal }}>\rho_{\mathrm{THR}} \\
p 53_{\mathrm{THR}}^{\mathrm{low}} \text { for } \rho_{\text {normal }} \leq \rho_{\mathrm{THR}}
\end{array} .\right.
$$

Here, $\rho_{\text {normal }}$ for a normal cell at location $i$ is given by

$$
\rho_{\text {normal }}(i)=\frac{\sum_{k \in \Theta_{i}} \# \text { normal cells at site } k}{\sum_{k \in \Theta_{i}} \# \text { normal }+\# \text { cancer cells at site } k},
$$

where the neighbourhood $\Theta_{i}$ is simply the cell's lattice site $i$ if that site contains more than one cell, and otherwise $\Theta_{i}$ includes nearest neighbouring lattice sites. Thus, 
normal cells are more likely to undergo apoptosis when sharing a site with or surrounded by cancer cells. This, and competition for nutrient, is a key mechanism for invasion.

Cancer cells enter (leave) quiescence when [p27] $>p 27_{e}\left([p 27]<p 27_{l}\right)$. Upon entering quiescence a timer $T_{\text {quiescent }}$ is set to zero and subsequently is incremented for each time step that the cell remains quiescent. Cancer cell apoptosis occurs when a cancer cell is quiescent for too long (i.e. when $T_{\text {quiescent }} \geq T_{\text {death }}$ ).

\section{A.3 Vascular flow and adaptation}

The flow in each vessel, indexed by $i$, is assumed to be laminar steady Poiseuille flow, i.e.

$$
\dot{Q}_{i}=\frac{\pi R_{i}^{4}}{8 \mu\left(R_{i}, H_{i}\right) L_{i}} \Delta P_{i}
$$

where $\dot{Q}_{i}$ is the flow rate in vessel $i, \Delta P_{i}$ is the pressure difference, and $\mu\left(R_{i}, H_{i}\right)=$ $\mu_{0} \mu_{\mathrm{rel}}\left(R_{i}, H_{i}\right)$ is a haematocrit and radius dependent blood viscosity [40], where $\mu_{0}$ is the plasma viscosity and

$$
\begin{aligned}
\mu_{0.45}(R) & =6 e^{-0.17 R}+3.2-2.44 e^{-0.06(2 R)^{0.645}} \\
C(R) & =\left(0.8+e^{-0.15 R}\right)\left(-1+\frac{1}{1+10^{-11}(2 R)^{12}}\right)+\frac{1}{1+10^{-11}(2 R)^{12}} \\
\mu_{\text {rel }}(R, H) & =\left[1+\left(\mu_{0.45}(R)-1\right) \frac{(1-H)^{C}-1}{(1-0.45)^{C}-1}\left(\frac{2 R}{2 R-1.1}\right)^{2}\right]\left(\frac{2 R}{2 R-1.1}\right)^{2} .
\end{aligned}
$$

At each node, except for inflows and outflows, conservation of mass means that the total flow into and out of each node must balance. Using Eq. (18) to write those flows in terms of pressures, together with specified inflow and outflow pressures $\left(P_{\text {in }}\right.$ and $\left.P_{\text {out }}\right)$, yields a set of linear equations for the pressures at each node. Given the nodal pressures, Eq. (18) is used to calculate the flow in each vessel segment.

Given the flows $\dot{Q}_{i}$ and vessel radii $R_{i}$, the inflow haematocrit, $H_{\text {in }}$ can be propagated through the vessel network. Asymmetric haematocrit splitting can be implemented as described in [14], where the degree of asymmetry is parameterised by $\alpha$. However, as noted in Sect. 2.1, we assume symmetric splitting in this paper (by setting $\alpha=0$ ) in order to focus on the dynamics of pruning and angiogenesis.

Updated haematocrit values can then be fed into the calculation of vessel radii adaptation defined by Eqs. (1)-(4), where the WSS set point is given by

$$
\tau(P)=100-86 \exp \left(-5000[\log (\log P)]^{5.4}\right) .
$$


The new vessel radii and haematocrit values will modify the flow calculation, so the sequence (calculate flow, haematocrit and radii) is iterated, with time step $\varepsilon_{t}$, until the maximum proportional change in vessel radius across the network is less than $R_{\text {reltol }}$. The default parameter set for the vasculature is given in Table 3.

\section{Appendix B: Parameter values}

Here we list all the parameter values for the simulations of Figs. 3 and 5a. All other figures use the same parameter set except for variations in initial vessel and/or cell configurations, maximum sprouting probability $P_{\text {sprout }}^{\max }$, oxygen permeability $\mathscr{P}_{c}$, chemotactic sensitivity $\gamma$, and VEGF induced vessel dilation $k_{m}^{V}$. The altered parameter values are indicated in the relevant figure legends.

Table 1 Existing parameters for the multiscale model, common to the whole simulation

\begin{tabular}{ll}
\hline Parameter & Default value \\
\hline$\Delta t$ & $30(\mathrm{~min})$ \\
$\Delta x$ & $40(\mu \mathrm{m})$ \\
Domain size & $50 \times 50$ cells \\
{$[C d h 1]_{0}$} & 0.9 \\
{$[c y c C D K]_{0}$} & 0.01 \\
$M_{0}$ & 5.0 \\
{$[p 27]_{0}$} & 0.0 \\
{$[n p R B]_{0}$} & 0.0 \\
$b_{1}$ & $1.0\left(\mathrm{~min}^{-1}\right)$ \\
$b_{3}$ & $10.0\left(\mathrm{~min}^{-1}\right)$ \\
$J_{3}$ & 0.04 \\
$b_{4}$ & $35.0\left(\mathrm{~min}^{-1}\right)$ \\
$J_{4}$ & 0.04 \\
$a_{4}$ & $0.04\left(\mathrm{~min}^{-1}\right)$ \\
$a_{2}$ & $1.0\left(\mathrm{~min}^{-1}\right)$ \\
$a_{3}$ & $0.25\left(\mathrm{~min}^{-1}\right)$ \\
$\eta$ & $0.005\left(\mathrm{~min}^{-1}\right)$ \\
$M^{*}$ & 10.0 \\
$c_{2}$ & $0.01\left(\mathrm{~min}^{-1}\right)$ \\
$B$ & 0.01 \\
$d_{2}$ & $0.1\left(\mathrm{~min}^{-1}\right)$ \\
$d_{1}$ & $0.01\left(\mathrm{~min}^{-1}\right)$ \\
{$[p 53]_{0}$} & 0.0 \\
$k_{7}$ & 0.0 \\
$k_{7}^{\prime}$ & $0.002\left(\mathrm{~min}^{-1}\right)$ \\
\hline & $0.01\left(\mathrm{~min}^{-1}\right)$ \\
\hline &
\end{tabular}


Table 1 continued

\begin{tabular}{ll}
\hline Parameter & \multicolumn{1}{c}{ Default value } \\
\hline$C_{p 53}$ & 0.01 \\
$k_{8}$ & $0.002\left(\mathrm{~min}^{-1}\right)$ \\
$J_{5}$ & 0.04 \\
$k_{8}^{\prime}$ & $0.01\left(\mathrm{~min}^{-1}\right)$ \\
$C_{\text {VEGF }}$ & 0.01 \\
$\mathscr{P}_{c}$ & 700 \\
$\mathscr{P}_{v}$ & 11,400 \\
$\delta_{v}$ & 10.0 \\
$D_{c}$ & 0.00145 \\
$D_{v}$ & 0.00145 \\
$R_{\mathrm{ex}}$ & 5 \\
\hline
\end{tabular}

See Sect. 2.1, Appendix A and [2,4,5,14]. The parameters for Eqs. (9)-(15) are listed in their order of appearance

Table 2 Parameter values that differ for normal and cancer cells, where a dash indicates a parameter that is not defined for that cell type

\begin{tabular}{|c|c|c|}
\hline Parameter & Normal cell & Cancer cell \\
\hline$a_{1}$ & $0.05\left(\min ^{-1}\right)$ & $0.4\left(\min ^{-1}\right)$ \\
\hline$c_{1}$ & $0.1\left(\min ^{-1}\right)$ & $0.007\left(\mathrm{~min}^{-1}\right)$ \\
\hline$\chi$ & 1.0 & 0.0 \\
\hline$k_{8}^{\prime \prime}$ & $-0.002\left(\min ^{-1}\right)$ & $0.002\left(\mathrm{~min}^{-1}\right)$ \\
\hline$\rho_{\mathrm{THR}}$ & 0.75 & - \\
\hline$D_{m}$ & 1 & 1 \\
\hline$C d h 1_{\mathrm{THR}}$ & 0.004 & 0.05 \\
\hline$c y c C D K_{\mathrm{THR}}$ & 0.2 & 0.05 \\
\hline$p 53_{\text {THR }}^{\text {high }}$ & 0.8 & - \\
\hline$p 53_{\mathrm{THR}}^{\text {low }}$ & 0.08 & - \\
\hline$T_{\text {death }}$ & - & 4,000 (min) \\
\hline$p 27_{e}$ & - & 1.05 \\
\hline$p 27_{l}$ & - & 1.0 \\
\hline$V_{\mathrm{THR}}$ & 0.27 & - \\
\hline$k_{v}(\mathbf{x})$ & 0.3 & 0.3 \\
\hline
\end{tabular}

See Sect. 2.1, Appendix A.3 and [2,4,5,14]. Parameters in Eqs. (9)-(15) are listed in their order of appearance 
Table 3 Vasculature parameters, as described in Sect. 2.1, Appendix A.3 and [2,4,5,14]

\begin{tabular}{|c|c|c|}
\hline Parameter & Default & \\
\hline$P_{\text {in }}$ & $25(\mathrm{mmHg})$ & \\
\hline$P_{\text {out }}$ & $15(\mathrm{mmHg})$ & \\
\hline$H_{\text {in }}$ & 0.45 & \\
\hline$\mu_{0}$ & $1.2\left(\mathrm{~g} \mathrm{~cm} \mathrm{~min}^{-2}\right)$ & \\
\hline$\alpha$ & 0.0 & \\
\hline$k_{s}$ & $1.15\left(\mathrm{~s}^{-1}\right)$ & \\
\hline$k_{p}$ & $1.0\left(\mathrm{~s}^{-1}\right)$ & \\
\hline$k_{m}^{0}$ & $1 . \dot{6}\left(\mathrm{~s}^{-1}\right)$ & \\
\hline$k_{m}^{V}$ & 0.0 & \\
\hline$V_{0}$ & $10^{-3}$ & \\
\hline$\tau_{\text {ref }}$ & 0 & \\
\hline$\dot{Q}_{\text {ref }}$ & $4 \times 10^{-5}\left(\mathrm{~cm}^{3} \mathrm{~min}^{-1}\right)$ & \\
\hline$\epsilon_{t}$ & $10^{-4}(\mathrm{~s})$ & \\
\hline$R_{\mathrm{MIN}}$ & $1(\mu \mathrm{m})$ & \\
\hline$R_{\mathrm{MAX}}$ & $50(\mu \mathrm{m})$ & \\
\hline$R_{\text {reltol }}$ & $10^{-4}$ & \\
\hline$\tau_{w}^{\text {crit }}$ & $8 . \dot{3}\left(\right.$ dynes $\left.\mathrm{cm}^{-2}\right)$ & $\begin{array}{l}\text { New: wall shear stress } \\
\text { threshold for pruning }\end{array}$ \\
\hline$T_{\text {prune }}$ & $4,000(\min )$ & $\begin{array}{l}\text { New: time a vessel can } \\
\text { survive low WSS }\end{array}$ \\
\hline
\end{tabular}

New parameters for vessel pruning are indicated—see Sect. 2.1

Table 4 New parameters for angiogenic sprouting, or existing parameters that extend to endothelial cells in addition to normal and cancer cells

\begin{tabular}{llll}
\hline Parameter & Normal & Cancer & Endothelial cell \\
\hline$k(\mathbf{x})$ & 13 & 20 & 5 \\
$D$ & 0 & $53 . \dot{3}\left(\mu \mathrm{m}^{2} \mathrm{~min}^{-1}\right)$ & $53 . \dot{3}\left(\mu \mathrm{m}^{2} \mathrm{~min}^{-1}\right)$ \\
$\gamma$ & 0 & 0 & $8 \times 10^{4}(\mu \mathrm{m})$ \\
$N_{m}$ & 1 & 1 & 2 \\
$E_{m}$ & - & - & 2 \\
$P_{\text {sprout }}^{\max }$ & - & - & $0 . \dot{3} \times 10^{-3}\left(\mathrm{~min}^{-1}\right)$ \\
$V_{\text {sprout }}$ & - & - & 0.001 \\
$M$ & - & 50 & 10 \\
\hline
\end{tabular}

See Sect. 2.2, Eqs. (5), (7) and (8), and Appendix A

\section{References}

1. Addison-Smith B, McElwain S, Maini PK (2008) A simple mechanistic model of sprout spacing in tumour-associated angiogenesis. J Theor Biol 250(1):1-15

2. Alarcón T, Byrne HM, Maini PK (2003) A cellular automaton model for tumour growth in an inhomogeneous environment. J Theor Biol 225:257-274 
3. Alarcón T, Byrne HM, Maini PK (2004) A mathematical model of the effects of hypoxia on the cellcycle of normal and cancer cells. J Theor Biol 229(3):395-411

4. Alarcón T, Byrne HM, Maini PK (2005) A multiple scale model for tumor growth. Multiscale Model Sim 3:440-475

5. Alarcón T, Owen MR, Byrne HM, Maini PK (2006) Multiscale modelling of tumour growth and therapy: the influence of vessel normalisation on chemotherapy. Comput Math Methods Med 7(2-3): 85-119

6. Anderson ARA (2005) A hybrid mathematical model of solid tumour invasion: the importance of cell adhesion. Math Med Biol 22:163-186

7. Anderson ARA, Chaplain MAJ (1998) Continuous and discrete mathematical models of tumorinduced angiogenesis. Bull Math Biol 60(5):857-899

8. Arakelyan L, Merbl Y, Agur Z (2005) Vessel maturation effects on tumour growth: validation of a computer model in implanted human ovarian carcinoma spheroids. Eur J Cancer 41(1):159-167

9. Arakelyan L, Vainstein V, Agur Z (2002) A computer algorithm describing the process of vessel formation and maturation, and its use for predicting the effects of anti-angiogenic and anti-maturation therapy on vascular tumor growth. Angiogenesis 5(3):203-214

10. Balding D, McElwain DLS (1985) A mathematical model of tumor induced capillary growth. J Theor Biol 114:55-73

11. Bartha K, Rieger H (2006) Vascular network remodeling via vessel cooption, regression and growth in tumors. J Theor Biol 241(4):903-918

12. Bentley K, Gerhardt H, Bates PA (2008) Agent-based simulation of Notch-mediated tip cell selection in angiogenic sprout initialisation. J Theor Biol 250(1):25-36

13. Bertuzzi A, Gandolfi A (2000) Cell kinetics in a tumour cord. J Theor Biol 204(4):587-599

14. Betteridge R, Owen MR, Byrne HM, Alarcón T, Maini PK (2006) The impact of cell crowding and active cell movement in vascular tumour growth. Netw Heterog Media 1:515-535

15. Byrne HM, Alarcón T, Owen MR, Webb SD, Maini PK (2006) Modelling aspects of cancer dynamics: a review. Philos Trans R Soc A 364:1563-1578

16. Byrne HM, Chaplain MAJ (1995) Mathematical models for tumour angiogenesis: numerical simulations and nonlinear wave solutions. Bull Math Biol 57(3):461-486

17. Carmeliet P (2003) Angiogenesis in health and disease. Nat Med 9:653-660

18. Chaplain MAJ, McDougall SR, Anderson ARA (2006) Mathematical modeling of tumor-induced angiogenesis. Ann Rev Biomed Eng 8:233-257

19. Clark ER (1918) Studies on the growth of blood-vessels in the tail of the frog larva-by observation and experiment on the living animal. Am J Anat 23(1):37-88

20. Dorman S, Deutsch A (2002) Modeling of self-organized avascular tumor growth with cellular automata. In Silico Biol 2:35

21. Folkman J (1971) Tumour angiogenesis-therapeutic implications. New Engl J Med 285:1182-1186

22. Folkman J, Klagsburn M (1987) Angiogenic factors. Science 235:442-447

23. Gevertz JL, Torquato S (2006) Modeling the effects of vasculature evolution on early brain tumor growth. J Theor Biol 243(4):517-531

24. Hellstrom M, Phng LK, Hofmann JJ, Wallgard E, Coultas L, Lindblom P, Alva J, Nilsson AK, Karlsson L, Gaiano N, Yoon K, Rossant J, Iruela-Arispe ML, Kalen M, Gerhardt H, Betsholtz C (2007) Dll4 signalling through Notch1 regulates formation of tip cells during angiogenesis. Nature 445(7129):776-780

25. Jain RK (1987) Determinants of tumour blood flow: a review. Cancer Res 47:2461-2468

26. Jain RK (2003) Molecular regulation of vessel maturation. Nat Med 9:685-693

27. Jain RK (2005) Normalization of tumour vasculature: an emerging concept in antiangiogenic therapy. Science 307:58-62

28. Lee DS, Rieger H, Bartha K (2006) Flow correlated percolation during vascular remodeling in growing tumors. Phys Rev Lett 96(5):058,104-4

29. Levine HA, Sleeman BD, Nilsen-Hamilton M (2001) Mathematical modeling of the onset of capillary formation initiating angiogenesis. J Math Biol 42(3):195-238

30. Levine HA, Tucker AL, Nilsen-Hamilton M (2002) A mathematical model for the role of cell signal transduction in the initiation and inhibition of angiogenesis. Growth Factors 20(4):155-175

31. Mantzaris N, Webb SD, Othmer HG (2004) Mathematical modelling of tumour angiogenesis: a review. J Math Biol 49:111-187 
32. McDougall SR, Anderson ARA, Chaplain MAJ (2006) Mathematical modelling of dynamic adaptive tumour-induced angiogenesis: clinical implications and therapeutic targeting strategies. J Theor Biol 241(3):564-89

33. McDougall SR, Anderson ARA, Chaplain MAJ, Sherratt JA (2002) Mathematical modelling of flow through vascular networks: implications for tumour-induced angiogenesis and chemotherapy strategies. Bull Math Biol 64(4):673-702

34. Meeson A, Palmer M, Calfon M, Lang R (1996) A relationship between apoptosis and flow during programmed capillary regression is revealed by vital analysis. Development 122(12):3929-3938

35. Othmer HG, Stevens A (1997) Aggregation, blowup, and collapse: the ABC's of taxis in reinforced random walks. SIAM J Appl Math 57(4):1044-1081

36. Owen MR, Byrne HM, Lewis CE (2004) Mathematical modelling of the use of macrophages as vehicles for drug delivery to hypoxic tumour sites. J Theor Biol 226:377-391

37. Patel A, Gawlinski E, Lemieux S, Gatenby R (2001) A cellular automaton model of early tumor growth and invasion: the effects of native tissue vascularity and increased anaerobic tumor metabolism. J Theor Biol 213:315-331

38. Plank M, Sleeman B (2004) Lattice and non-lattice models of tumour angiogenesis. Bull Math Biol 66(6):1785-1819

39. Plank MJ, Sleeman BD (2003) A reinforced random walk model of tumour angiogenesis and antiangiogenic strategies. Math Med Biol 20(2):135-181

40. Pries A, Reglin B, Secomb T (2001) Structural adaptation of microvascular networks: functional roles of adaptive responses. Am J Physiol 281:H1015-H1025

41. Pugh C, Rattcliffe P (2003) Regulation of angiogenesis by hypoxia: role of the HIF system. Nat Med 9:677-684

42. Resnick N, Yahav H, Shay-Salit A, Shushy M, Schubert S, Zilberman LCM, Wofovitz E (2003) Fluid shear stress and the vascular endothelium: for better and for worse. Progress Biophys Mol Biol 81(3):177-199

43. Risau W (1997) Mechanisms of angiogenesis. Nature 386:871-875

44. Siekmann AF, Lawson ND (2007) Notch signalling limits angiogenic cell behaviour in developing zebrafish arteries. Nature 445(7129):781-784

45. Stephanou A, McDougall SR, Anderson ARA, Chaplain MAJ (2005) Mathematical modelling of flow in $2 \mathrm{~d}$ and $3 \mathrm{~d}$ vascular networks: applications to anti-angiogenic and chemotherapeutic drug strategies. Math Comput Modell 41(10):1137-1156

46. Stephanou A, McDougall SR, Anderson ARA, Chaplain MAJ (2006) Mathematical modelling of the influence of blood rheological properties upon adaptative tumour-induced angiogenesis. Math Comput Modell 44(1-2):96-123

47. Stokes CL, Lauffenburger DA (1991) Analysis of the roles of microvessel endothelial cell random motility and chemotaxis in angiogenesis. J Theor Biol 152(3):377-403

48. Suchting S, Freitas C, le Noble F, Benedito R, Breant C, Duarte A, Eichmann A (2007) The Notch ligand Delta-like 4 negatively regulates endothelial tip cell formation and vessel branching. Proc Natl Acad Sci 104(9):3225-3230

49. Teicher B (1996) A systems approach to cancer therapy. Cancer Metastasis Rev 15:247-272

50. Tong R, Boucher Y, Kozin S, Winkler F, Kicklin D, Jain R (2004) Vascular normalisation by VEGFR2 blockade induces a pressure gradient accros the vasculature and improves drug penetration in tumours. Cancer Res 64:3731-3736

51. Tyson JJ, Novák B (2001) Regulation of the eukaryotic cell-cycle: molecular anatagonism, hysteresis, and irreversible transitions. J Theor Biol 210:249-263

52. Welter M, Bartha K, Rieger H (2008) Emergent vascular network inhomogeneities and resulting blood flow patterns in a growing tumor. J Theor Biol 250(2):257-280

53. Winkler F, Kozin S, Tong R, Chae S-S, Booth M, Garkavtsev I, Xu L, Hicklin D, Fukumura D, di Tomaso E, Munn L, Jain R (2004) Kinetics of vascular normalisation by VEGFR2 blockade governs brain tumour response to radiation: role of oxyxgenation, angiopoietin-1 and matrix metalloprotienases. Cancer Cell 6:553-563

54. Yen RT, Fung YC (1978) Effect of velocity distribution on red cell distribution in capillary blood vessels. AJP Heart Circ Physiol 235(2):H251-H257 